Published in "Transportation Research Part B: Methodological", 2018, vol. 114, pp. 213-240, which should be cited to refer to this work.

DOI : $10.1016 / j . t r b .2018 .05 .015$

\title{
A Unified Framework for Rich Routing Problems with Stochastic Demands
}

\author{
Iliya Markov ${ }^{\mathrm{a},}{ }^{,}$, Michel Bierlaire ${ }^{\mathrm{a}}$, Jean-François Cordeau ${ }^{\mathrm{b}}$, \\ Yousef Maknoon ${ }^{\mathrm{c}}$, and Sacha Varone ${ }^{\mathrm{d}}$ \\ $\mathrm{a}_{\text {Transport and Mobility Laboratory }}$ \\ School of Architecture, Civil and Environmental Engineering \\ École Polytechnique Fédérale de Lausanne \\ Station 18, 1015 Lausanne, Switzerland \\ Email: \{iliya.markov, michel.bierlaire\}@epfl.ch \\ ${ }^{\mathrm{b}}$ CIRRELT and HEC Montréal \\ 3000 chemin de la Côte-Sainte-Catherine \\ Montréal, Canada H3T 2A7 \\ Email: jean-francois.cordeau@hec.ca \\ ${ }^{\mathrm{c}}$ Faculty of Technology, Policy, and Management \\ Delft University of Technology \\ Jaffalaan 5, 2628 BX Delft, The Netherlands \\ Email: m.y.maknoon@tudelft.nl \\ ${ }^{\mathrm{d}}$ Haute École de Gestion de Genève \\ University of Applied Sciences Western Switzerland (HES-SO) \\ Campus Battelle, Rue de la Tambourine 17, 1227 Carouge, Switzerland \\ Email: sacha.varone@hesge.ch
}

February 27, 2018

\begin{abstract}
We introduce a unified framework for rich vehicle and inventory routing problems with complex physical and temporal constraints. Demands are stochastic, can be non-stationary, and are forecast using any model that provides the expected demands and their error term distribution, which can be any theoretical or empirical distribution. We offer a detailed discussion on the modeling of demand stochasticity, focusing on the probabilities and cost effects of undesirable events, such as stock-outs, breakdowns and route failures, and their associated recourse actions. Tractability is achieved through the ability to pre-compute or at least partially pre-process the stochastic information, which is possible under mild assumptions for a general inventory policy. We integrate the stochastic aspect into a mixed integer non-linear program, illustrate applications to various problem classes, and show how to model specific problems through the lens of inventory routing. The case study is based on two sets of realistic instances, representing a waste collection inventory routing problem and a facility maintenance problem, respectively. We analyze the effects of our assumptions on modeling realism and tractability, and demonstrate that our framework significantly outperforms deterministic policies in its ability to limit the number of undesirable events for the same routing cost.
\end{abstract}

Keywords: unified framework; rich routing problem; stochastic demand; forecasting; tractability; recourse

* Corresponding author 


\section{Introduction}

The Vehicle Routing Problem (VRP) is an integer programming and combinatorial optimization problem that seeks to find the cheapest set of tours to serve a number of customers. In its basic form, there is a single depot that accommodates a homogeneous fleet and each vehicle performs a single tour that starts and ends at the depot. Customers have fixed demands of a single commodity and the number of customers in each tour is only limited by the vehicle capacity. The VRP was formally introduced in the seminal work of Dantzig and Ramser (1959) in the context of fuel delivery and is one of the most practically relevant and widely studied problems in operations research. A generalization of the VRP, the Inventory Routing Problem (IRP) introduces a planning horizon and seeks to optimize simultaneously the vehicle tours, delivery times and delivery quantities. The seminal work on the IRP was motivated by the delivery and inventory management of industrial gases (Bell et al., 1983). The literature on the VRP, the IRP and their many variants is vast, driven both by their mathematical properties and by their numerous practical applications in the distribution and collection of goods and the transportation of people. The need to solve ever larger and richer routing problems has pushed researchers over the past decades to develop advanced modeling techniques and solution methodologies.

In this context, rich routing problems are generalizations of the basic VRP that include a variety of practically relevant features. For instance, the fleet may be heterogeneous instead of homogeneous. Each vehicle may perform multiple tours per day, instead of one, and visit both customers and replenishment stops subject to time windows and accessibility restrictions. Depending on the application, there could be multiple depots with the possibility of open tours that have different origin and destination depots or multi-day tours that last over several days. Driving schedules must respect regulations on maximum working hours while equity considerations might imply that all drivers work similar hours. Customers may have preferences for a given driver or visit periodicity. Because of their inherent difficulty, such problems have seen increased academic interest in recent years due to the methodological and technological progress that has been made (Lahyani et al. 2015). Another defining characteristic of real-world problems is uncertainty, which presents itself in the form of stochastic demands, stochastic customer presence, stochastic travel and service times, etc (Gendreau et al., 2016). Rich routing features inevitably compound the effects of the uncertainty associated with these stochastic parameters. Failure to account for uncertainty often leads to solutions that are suboptimal or even infeasible (Louveaux, 1998).

Deviation of the stochastic parameters from their expected values often leads to the occurrence of undesirable events. Longer than expected travel or service times may result in the inability to serve subsequent customers within their time windows. Higher than expected demands may lead to customer stock-outs and to route failures. A route failure occurs when a delivery vehicle runs out of capacity before its next replenishment stop (Dror and Trudeau, 1986). These undesirable events often require corrective action, referred to as recourse. The dispatch of an emergency delivery vehicle is an example of recourse in the case of stock-out. A detour to a replenishment stop can be performed in the case of route failure. Given that undesirable events and their recourse actions are expensive, good solutions should limit their number.

In this work, we propose a unified framework for modeling and solving rich routing problems, including among others the VRP and the IRP, in the presence of non-stationary stochastic demands. Our contribution is four-fold and starts with the explicit modeling of the probabilities and cost effects of undesirable events and their associated recourse actions for a generic rich routing problem. This is achieved through the use of dynamic probabilistic information in the objective function, which alleviates the curse of dimensionality that plagues many scenario-based approaches. Our approach is oriented towards cost minimization and the pricing of uncertainty, as would be the case for a cost-minimizing firm. As such, we distinguish it from robust optimization (Bertsimas and Sim, 2003, 2004) where the focus is on protecting feasibility.

Our second contribution concerns the integration of real-world demand forecasting techniques. The routing literature, and the IRP literature in particular, typically uses simple forecasting techniques, if at all. Besides, stochastic demands are usually modeled as independent and identically distributed (iid) random variables from the normal distribution (Gendreau et al., 2016). Our framework can use any state-of-the-art forecasting model that provides the expected demands over the planning horizon and a measure of uncertainty represented by their error term distribution. The latter can be any theoretical or empirical distribution, thus 
addressing an important gap between theory and practice Gendreau et al. (2016). This leads us to the third contribution, which concerns the preservation of computational tractability, given the above generalizations and the presence of rich routing features. Using simulation techniques, we can pre-compute or at least partially pre-process the bulk of the probabilistic information under mild assumptions for a general inventory policy.

Our final contribution lies in the generality and practical relevance of the approach. We develop a Mixed Integer Non-Linear Program (MINLP) and illustrate applications to rich routing problems borrowed from the fields of health care, maritime operations, waste collection, facility maintenance, and others. Moreover, we demonstrate that conceptually different problems like the waste collection IRP and the facility maintenance problem fit the same modeling framework. Thus, our results and conclusions can potentially be generalized to other contexts as well. The case study is based on two sets of realistic instances, representing a waste collection IRP and a facility maintenance problem, respectively. We analyze the effects of our assumptions on modeling realism and tractability, and demonstrate that our framework significantly outperforms alternative deterministic policies in its ability to limit the number of undesirable events for the same routing cost.

The remainder of this article is organized as follows. Section 2 offers a brief review of the relevant literature on rich routing problems from various application fields with a focus on demand stochasticity. Section 3 introduces the main concepts and modeling elements used by the unified framework. These are further discussed and elaborated in Section 4, which details the treatment of demand stochasticity, and Section 5 . which develops the optimization model. In turn, Section 6 provides examples of adapting the framework to the various application fields. Section 7 presents the numerical experiments and, finally, Section 8 concludes and outlines future work directions.

\section{Related Literature}

This section offers a review of routing problems with stochastic demands, starting from rich vehicle and inventory routing problems in general and then exploring several specific and pertinent application fields. The analysis comments on the variety of approaches used in integrating stochastic demands in the modeling and solution process, thus highlighting the need for a unified approach.

\subsection{Rich Vehicle and Inventory Routing Problems}

Rich vehicle routing problems are multi-constrained routing problems that extend the classical capacitated VRP (Dantzig and Ramser, 1959) by including features relevant to real-world problems. The recent work of Lahyani et al. (2015) develops a taxonomy and definition of rich VRPs. Surveys on various aspects concerning heterogeneous fleets, intermediate replenishment facilities, time windows, open tours and multiple depots are available in Markov et al. (2014, 2016b) and Markov et al. (2016a). Rich routing problems often include an uncertainty component. In dynamic problems, parameters are partly unknown and gradually revealed with time. In dynamic and stochastic problems, we have in addition access to probability information related to the unknown parameters. Ritzinger et al. (2016) summarize the recent literature on dynamic and stochastic VRPs and offer a classification scheme based on the available stochastic information. Gendreau et al. (2016) center their survey on the state of the art of the a priori and the re-optimization paradigms for stochastic VRPs, the two being the predominantly used paradigms by researchers.

Although multi-constrained IRPs with real-world features have recently begun to appear in the literature, the term rich IRP has not established itself as in the case of the VRP. Zhalechian et al. (2016) and Soysal (2016), for example, discuss closed-loop IRP systems with stochastic demands. Both include environmental considerations in the objective function. Zhalechian et al. (2016) also include social considerations, present a fuzzy approach, and develop a hybrid meta-heuristic and a lower bounding procedure, which are applied on a small case study. Soysal (2016) use CPLEX to solve a small case study and, based on a simulation experiment, confirm the benefit of including uncertainty in the model. Rahimi et al. (2017) describe a rich IRP with environmental considerations and stochastic parameters, including stochastic demand, and propose 
a fuzzy approach. Their solution methodology relies on a meta-heuristic from the literature. However, the focus of their numerical experiments is not on the effect of uncertainty. Furthermore, none of these studies models explicitly recourse actions in the events of stock-outs and route failures, which occur as a consequence of demand uncertainty.

Our problem considers a finite planning horizon with possibly non-stationary distributions. Markov et al. (2016a) provide a review of the literature on road-based stochastic IRPs over a finite horizon and discuss the advantages and disadvantages of various modeling approaches. Sections 2.2, 2.3 and 2.4 below extend the survey to several additional application areas of routing problems with stochastic demands that can be modeled using the unified framework. Finally, Section 2.5 positions our approach. We note that other problems consider an infinite horizon and the focus is on optimizing a discounted cost or revenue modeled as a Markov decision process, usually assuming that probability distributions do not change over time. The solution methodology often relies on dynamic programming (Berman and Larson, 2001) or approximate dynamic programming (Campbell et al., 1998; Kleywegt et al., 2002, 2004:|Adelman, 2004). Other approaches include GRASP (Hvattum and Løkketangen, 2009) and scenario trees truncated over a finite planning horizon (Hvattum et al., 2009). Such problems are out of the scope of this research. More detailed surveys can be found in Coelho et al. (2014) and Andersson et al. (2010).

\section{$2.2 \quad$ Health Care Routing Problems}

Stochastic demand appears in health care routing problems involving the pick-up and delivery of drugs, biological samples, and medical equipment. Hemmelmayr et al. (2010) solve a stochastic blood distribution problem, which considers shortfalls and spoilage. To balance delivery and spoilage costs, they limit the probability of spoilage to $5 \%$ by sampling product usage during the spoilage period and taking the $5 \%$ quantile as the maximum inventory level at the hospital. Hemmelmayr et al. (2010) develop a two-stage stochastic program with recourse, assuming knowledge of the inventory in the beginning of each day of the planning horizon. The authors extend an exact approach and a VNS meta-heuristic from the literature, in both cases using external sampling to convert the two-stage stochastic optimization problem into a deterministic one. Through a simulation experiment, they show that a simple recourse policy is sufficient to provide a reliable and cost-efficient blood supply. Niakan and Rahimi (2015) and Shi et al. (2017) study the problem of delivering drugs with uncertain demands to patient homes. Both articles apply fuzzy programming approaches to the problem and report the added value of incorporating uncertainty into the model. The broader literature on health care routing problems identifies workload balancing and the continuity of service, or continuity of care in this specific context, as two of the most important concerns in this field (see e.g. Lanzarone and Matta, 2009, 2012, Lanzarone et al., 2012, Errarhout et al., 2014, 2016).

\subsection{Waste Collection Routing Problems}

Markov et al. (2016a) describe a stochastic IRP for the collection of recyclable waste with the integration of demand forecasting. Demand stochasticity leads to the occurrence of container overflows and route failures. The proposed stochastic model significantly outperforms alternative deterministic policies in its ability to limit the occurrence of container overflows for the same routing cost. Still in the area of waste collection, Johansson (2006) and Mes (2012) use simulation to confirm the benefits of migrating from static to dynamic collection policies in Malmö, Sweden and a study area in the Netherlands, respectively, where containers are equipped with level and motion sensors, respectively. Mes (2012) finds a positive added value of investing in level sensors compared to simple motion sensors that detect when a container is emptied. Mes et al. (2014) apply optimal learning techniques to tune the parameters related to inventory control (deciding which containers to select) assuming accurate container level information. Nolz et al. (2011) develop a tabu search algorithm for a stochastic IRP for the collection of infectious waste from pharmacies. Nolz et al. (2014b) propose a scenario sampling method and an ALNS algorithm for the same problem. Nolz et al. (2014a) extend this to a bi-objective problem, trading off satisfaction of pharmacies, local authorities and the minimization of public health risks against routing costs. They propose three meta-heuristic approaches for this problem. Bitsch (2012) develops a VNS for an IRP applied to the collection of recyclable waste in a 
Danish region. Waste level is stochastic and containers should be emptied so that the probability of overflow is six standard deviations away.

\subsection{Maritime Routing Problems}

Papageorgiou et al. (2014) identify three features that distinguish maritime from road-based IRPs, specifically: 1) the absence of a central depot, which entails multi-period open tours, 2) the long travel times and port operations, which prolong the planning horizon, and 3) the shorter succession of port visits, in comparison to the typically dozens of customer visits in road-based IRPs. Cheng and Duran (2004) solve a crude oil transportation problem with inventory management, integrating discrete event simulation and stochastic optimal control. The optimal control problem is formulated as a Markov decision process that incorporates travel time and demand uncertainty. $\mathrm{Yu}(2009)$ discusses a problem with multiple supply and demand ports, where the only stochastic element is the demand. It is formulated as a stochastic program and branch-and-price is used to solve medium-sized instances. Arslan and Papageorgiou (2015) study a maritime fleet renewal and deployment problem under demand and charter cost uncertainty, which determines the fleet size, mix, and deployment strategy to satisfy stochastic demands over the planning horizon. They solve the problem in a rolling horizon fashion using a stochastic programming look-ahead model, and explore the impact of different scenario trees with different recourse functions. Zheng and Chen (2016) propose a real option model to solve a fleet replacement model under demand and fuel price uncertainty. Monte Carlo simulation is used to find replacement probabilities in future years and the net present value of cost savings. The distribution of Liquefied Natural Gas (LNG) is a particularly important application area. Moraes and Faria (2016) study an LNG planning problem for an oil and gas company. They develop a two-stage stochastic linear model to address uncertainties related to the LNG demand and spot prices.

\subsection{Discussion}

The reviewed literature reveals a variety of approaches for capturing demand uncertainty. Authors use different simplifying assumptions and modeling techniques, with or without explicit recourse policies and penalties for the occurrence of undesirable events. All these approaches come with their benefits and limitations. Scenario generation and stochastic modeling based on Markov decision processes both lead to problems that suffer from the curse of dimensionality for realistic-size instances (Pillac et al., 2013). Approximate dynamic programming (Powell, 2011) helps alleviate some of the issues in the latter case. In their recent work, Rossi et al. (2017) also note the instance size limitations of dynamic programming in solving the bowser routing problem, a special version of the IRP, and propose heuristic approximations. Thus, while scenario-based approaches allow significant freedom in modeling undesirable events and recourse actions, they are computationally heavy for an already hard combinatorial problem like ours. Robust optimization does not suffer from this limitation but its focus is on protecting against the worst-case scenario. It maintains feasibility for a given budget of uncertainty, is distribution-free, and relies on specific reformulations depending on whether parameter uncertainty in the standard-form mathematical program appears column-wise (Soyster, 1973), row-wise (Bertsimas and Sim, 2003, 2004), or only in the right-hand side (Minoux, 2009). Yet, complications arise if there is inter-row dependency in the uncertainty on the right-hand side (see Delage and Iancu, 2015). We do not see this approach very often used for routing problems (Gendreau et al., 2016), but we should mention the works of Sungur et al. (2008) and Gounaris et al. (2013) who consider stochastic demands in a VRP context, and Aghezzaf (2008) and Solyalı et al. (2012) in an IRP context. Chance constrained approaches guarantee that a constraint will be satisfied with a given probability. These are appropriate if uncertainty appears row-wise and have typically been used to model route failures in vehicle routing problems with stochastic demands (see references in Gendreau et al., 2014). We highlight that the majority of the distribution-based approaches in the literature on routing problems assume iid normally distributed demands (Gendreau et al., 2016).

Using a set of key concepts and modeling elements, our framework provides the ingredients for modeling and solving rich routing problems with non-stationary stochastic demands. The approach distinguishes itself through several unifying features, namely 1) the applicability to various problem types, including among 
others rich VRPs and IRPs, 2) the integration of real-world demand forecasting with very few distributional assumptions, 3) the explicit modeling of undesirable events and recourse actions and their direct integration in the objective function or the constraints, 4) the tractability of the resulting framework through the ability to pre-compute or at least partially pre-process most of the stochastic information for a general inventory policy, and 5) the intuitive evaluation of the produced solution through simulation. Simulation is used both to measure the frequency of occurrence of undesirable events in the final solution and to evaluate how closely it models the real cost given the imposed assumptions and modeling simplifications.

\section{$3 \quad$ Key Concepts and Modeling Elements}

This section introduces the key concepts and modeling elements used in our framework as well as the relationships among them. For the sake of generality, consider a problem in a distribution context. We comment on the changes that apply to a collection or another context when needed. There is a planning horizon $\mathcal{T}=\{0, \ldots, u\}$ of discrete time periods, such as days or another appropriate level of discretization. Deliveries are performed by a heterogeneous fixed fleet $\mathcal{K}$, with each vehicle $k \in \mathcal{K}$ defined by a per-period deployment $\operatorname{cost} \varphi_{k}$, a unit-distance running $\operatorname{cost} \beta_{k}$, a unit-time running cost $\theta_{k}$, and a capacity $\Omega_{k}$. The fleet reduces to a homogeneous one if the values of these parameters are identical for all vehicles.

For each vehicle $k \in \mathcal{K}$ and period $t \in \mathcal{T}$, we are given a directed graph $\mathcal{G}_{k t}\left(\mathcal{N}_{k t}, \mathcal{A}_{k t}\right)$. The set $\mathcal{O}$ includes all origin and destination depots, where $\mathcal{O}_{k t}^{\prime} \subseteq \mathcal{O}$ is the set of origin depots for vehicle $k$ in period $t$ and $\mathcal{O}_{k t}^{\prime \prime} \subseteq \mathcal{O}$ is the set of destination depots for vehicle $k$ in period $t$. In addition, $\mathcal{P}$ is the set of demand points, $\mathcal{D}$ is the set of supply points, $\mathcal{N}_{k t}=\mathcal{O}_{k t}^{\prime} \cup \mathcal{O}_{k t}^{\prime \prime} \cup \mathcal{P} \cup \mathcal{D}$ is the set of all points potentially reachable by vehicle $k$ in period $t$, and $\mathcal{A}_{k t}=\left\{(i, j): \forall i, j \in \mathcal{N}_{k t}, i \neq j\right\}$ is the set of arcs connecting the latter. The set $\mathcal{D}$ contains a sufficient number of replications of each supply point to allow multiple visits by the same vehicle in the same period. The distance matrix is asymmetric, with $\pi_{i j}$ the length of arc $(i, j) \in \mathcal{A}_{k t}$, for any vehicle $k$ and period $t$. Vehicle $k$ can have a specific travel time matrix for each period $t$, where $\tau_{i j k t}$ is the travel time of vehicle $k$ on arc $(i, j) \in \mathcal{A}_{k t}$ in period $t$. Point $i \in \mathcal{O} \cup \mathcal{P} \cup \mathcal{D}$ presents a time window $\left[\lambda_{i}, \mu_{i}\right]$, where $\lambda_{i}$ and $\mu_{i}$ stand for the earliest and latest possible start-of-service time at that point. There is a maximum of one service for demand point $i$ per period. Start of service after $\mu_{i}$ is not allowed and if the vehicle arrives before $\lambda_{i}$, it has to wait. Service duration at point $i$ is denoted by $\delta_{i}$, with service durations in the set $\mathcal{O}$ being zero.

Each demand point $i \in \mathcal{P}$ has an inventory capacity of $\omega_{i}$, a visit cost of $\xi_{i}$, and an inventory holding cost of $\eta_{i}$. It is visited at most once per period, while the parameter $\nu_{i}$ specifies the minimum number of times it must be visited over the planning horizon. There is the option of imposing periodicity on the visits as well. The set $\mathcal{C}_{i}$ contains the visit period combinations for demand point $i$, and the binary constant $\alpha_{r t}$ denotes whether period $t$ belongs to visit period combination $r \in \mathcal{C}_{i}$ for demand point $i$. The binary flags $\alpha_{i k t}$ denote whether point $i \in \mathcal{P} \cup \mathcal{D}$ is accessible by vehicle $k$ in period $t$. They can also be used to express continuity of service, restricting the vehicle(s) that can visit demand point $i$.

In period $t$, demand point $i$ exhibits non-stationary stochastic demand $\rho_{i t}$. It is important to highlight that stochasticity refers to normal operations, and not to hazard or deep uncertainty (Gendreau et al., 2016). Demand stochasticity implies a probability of stock-out, one of two possible states for each demand point, which happens when its inventory becomes negative. Let $\sigma_{i t}=1$ denote that demand point $i$ is in a state of stock-out in period $t$ and let $\sigma_{i t}=0$ denote the opposite. Point $i$ incurs a stock-cost of $\chi_{i}$ for all $t \in \mathcal{T}$ where $\sigma_{i t}=1$. For $t \in \mathcal{T}$ where $\sigma_{i t}=1$ and no vehicle $k \in \mathcal{K}$ visits demand point $i$, an emergency delivery recourse action is applied with a cost of $\zeta_{i}$. We apply a limited back-order policy where a delivery must be performed in the same period $t$ in which a stock-out occurs. We can limit the probability of stock-out at the demand points to a maximum allowable level $\gamma^{\mathrm{DP}}$. Demand stochasticity and the probability of stock-out are further discussed in Sections 4.1 and 4.2 .

In the IRP, the two classical inventory policies are the Order-Up-to (OU) level policy and the Maximum Level (ML) policy (Bertazzi et al., 2002, Archetti et al. 2011). Under the former delivery is up to the capacity $\omega_{i}$, while under the latter the delivery quantity is part of the decisions. We consider a discretized ML policy, 
which is more general than the OU policy, but less general than the classical ML policy. In the discretized ML policy, the delivery quantity is still part of the decisions, but is chosen from a discrete set. Let the set $\mathcal{L}_{i}$ define for each demand point $i$ its allowable discrete inventory levels. For the case where $\mathcal{L}_{i}=\left\{\omega_{i}\right\}, \forall i \in \mathcal{P}$, the discretized ML policy reduces to the OU policy. The use of a discretized ML policy allows the tractable pre-processing of much of the probability information related to undesirable events. This topic is further discussed in Section 4.2 .

A tour executed by vehicle $k$ in period $t$ starts from an origin $o^{\prime} \in \mathcal{O}_{k t}^{\prime}$ and ends at a destination $o^{\prime \prime} \in \mathcal{O}_{k t}^{\prime \prime}$ and is a sequence of demand and supply point visits. The maximum tour duration of vehicle $k$ in period $t$ is denoted by $\mathrm{H}_{k t}$. If $\mathrm{H}_{k t}=0$, vehicle $k$ is not available in period $t$. A tour's origin and destination need

Table 1: Notations

\begin{tabular}{|c|c|c|c|}
\hline \multicolumn{4}{|l|}{ Sets } \\
\hline $\begin{array}{l}\mathcal{T} \\
\mathcal{O}_{k t}^{\prime} \\
\mathcal{P} \\
\mathcal{N}_{k t} \\
\mathcal{C}_{i} \\
\mathfrak{S}_{k} \\
\mathcal{S}_{t}\end{array}$ & $\begin{array}{l}\text { planning horizon }=\{0, \ldots, u\} \\
\text { set of origins for vehicle } k \text { in period } t \\
\text { set of demand points } \\
=\mathcal{O}_{k t}^{\prime} \cup \mathcal{O}_{k t}^{\prime \prime} \cup \mathcal{P} \cup \mathcal{D} \\
\text { set of visit period combinations for demand point } i \\
\text { set of trips executed by vehicle } k \\
\text { set of demand points in trip } \mathscr{S} \text { visited in period } t\end{array}$ & $\begin{array}{l}\mathcal{T}^{+} \\
\mathcal{O}_{k t}^{\prime \prime} \\
\mathcal{D} \\
\mathcal{K} \\
\mathcal{L}_{i} \\
\mathscr{S}\end{array}$ & $\begin{array}{l}\text { shifted planning horizon }=\{1, \ldots, u, u+1\} \\
\text { set of destinations for vehicle } k \text { in period } t \\
\text { set of supply points } \\
\text { set of vehicles } \\
\text { set of discrete levels for demand point } i \\
\text { a particular trip in } \mathfrak{S}_{k}\end{array}$ \\
\hline \multicolumn{4}{|c|}{ Parameters } \\
\hline $\begin{array}{l}\varphi_{k} \\
\beta_{k} \\
\theta_{k} \\
\Omega_{k} \\
\pi_{i j} \\
\tau_{i j k t} \\
\lambda_{i}, \mu_{i} \\
\delta_{i} \\
\omega_{i} \\
\xi_{i} \\
\eta_{i} \\
\nu_{i} \\
\alpha_{r t} \\
\alpha_{i k t} \\
\rho_{i t} \\
\varepsilon_{i t} \\
\sigma_{i t} \\
\chi_{i} \\
\zeta_{i} \\
\mathrm{H}_{k t} \\
\Theta \\
\psi \\
C_{\mathscr{S}} \\
\gamma^{\mathrm{DP}} \\
\gamma^{\mathrm{RF}}\end{array}$ & $\begin{array}{l}\text { per-period deployment cost of vehicle } k \text { (monetary) } \\
\text { unit-distance running cost of vehicle } k \text { (monetary) } \\
\text { unit-time running cost of vehicle } k \text { (monetary) } \\
\text { capacity of vehicle } k \\
\text { length of arc }(i, j) \\
\text { travel time of vehicle } k \text { on arc }(i, j) \text { in period } t \\
\text { lower and upper time window bound at point } i \\
\text { service duration at point } i \\
\text { inventory capacity of demand point } i \\
\text { visit cost to demand point } i \text { (monetary) } \\
\text { inventory holding cost at demand point } i \text { (monetary) } \\
\text { minimum number of times that demand point } i \text { must } \\
1 \text { if period } t \text { belongs to visit period combination } r, 0 \\
1 \text { if point } i \text { is accessible by vehicle } k \text { in period } t, 0 \text { ot } \\
\text { stochastic demand of point } i \text { in period } t \\
\text { stochastic error term of demand point } i \text { in period } t \\
1 \text { if demand point } i \text { is in a state of stock-out in perio } \\
\text { stock-out cost at demand point } i \text { (monetary) } \\
\text { emergency delivery cost to demand point } i \text { (monetar } \\
\text { maximum tour duration for vehicle } k \text { in period } t \\
\text { penalty on the difference between the min and max } \\
\text { Route Failure Cost Multiplier (RFCM) } \in[0,1] \\
\text { the average routing cost of going from } \mathscr{S} \in \mathfrak{S}_{k} \text { to th } \\
\text { maximum allowable probability of stock-out at the d } \\
\text { maximum allowable probability of route failure in th }\end{array}$ & $\begin{array}{l}\text { ehicle } \\
\text { near } \\
\text { emanc } \\
\text { rang }\end{array}$ & $\begin{array}{l}\text { orkload over the planning horizon (monetary) } \\
\text { supply point and back to } \mathscr{S} \text { (monetary) } \\
\text { oint in the range of }(0,1] \\
\mathrm{f}(0,1]\end{array}$ \\
\hline \multicolumn{4}{|c|}{ Decision Variables } \\
\hline $\begin{array}{l}x_{i j k t} \\
y_{i k t} \\
z_{k t} \\
c_{i r} \\
\ell_{i r t} \\
q_{i k t} \\
Q_{i k t} \\
I_{i t} \\
S_{i k t} \\
\underline{b}_{k t}, \bar{b}_{k t} \\
\underline{B}, \bar{B}\end{array}$ & $\begin{array}{l}1 \text { if vehicle } k \text { traverses arc }(i, j) \text { in period } t, 0 \text { otherw } \\
1 \text { if point } i \text { is visited by vehicle } k \text { in period } t, 0 \text { other } \\
1 \text { if vehicle } k \text { is used in period } t, 0 \text { otherwise (binary) } \\
1 \text { if visit period combination } r \text { is assigned to demand } \\
1 \text { if discrete level } r \text { is chosen for demand point } i \text { in p } \\
\text { expected delivery quantity to demand point } i \text { by veh } \\
\text { expected cumulative quantity delivered by vehicle } k \\
\text { expected inventory at demand point } i \text { at the start of } \\
\text { start-of-service time of vehicle } k \text { at point } i \text { in period } \\
\text { lower and upper bound on the tour duration of vehic } \\
\text { lower and upper bound on the workload for each veh }\end{array}$ & $\begin{array}{l}\text { ise }(\mathrm{b} \\
\text { wise } \\
\text { point } \\
\text { eriod } \\
\text { icle } k \\
\text { arrivir } \\
\text { perio } \\
t(\text { con } \\
\text { le } k \text { ir } \\
\text { icle }(c\end{array}$ & $\begin{array}{l}\text { ry) } \\
\text { lary) } \\
0 \text { otherwise (binary) } \\
\text { otherwise (binary) } \\
\text { period } t \text { (continuous) } \\
\text { at point } i \text { in period } t \text { (continuous) } \\
\text { i.e. before delivery (continuous) } \\
\text { uous) } \\
\text { eriod } t \text { (continuous) } \\
\text { tinuous) }\end{array}$ \\
\hline
\end{tabular}


not coincide, and the correct definition of the sets $\mathcal{O}_{k t}^{\prime}$ and $\mathcal{O}_{k t}^{\prime \prime}$ implies that $\mathcal{O}_{k t}^{\prime \prime} \cap \mathcal{O}_{k(t+1)}^{\prime} \neq \emptyset$, i.e there is at least one depot where vehicle $k$ can end its tour in period $t$ and start its tour in period $t+1$. The correct definition of the above sets also implies that when $\mathrm{H}_{k t}=0, \exists o^{\prime} \in \mathcal{O}_{k t}^{\prime}$ and $o^{\prime \prime} \in \mathcal{O}_{k t}^{\prime \prime}$ s.t. $\pi_{o^{\prime} o^{\prime \prime}}=0$, i.e there is at least one physical depot at which vehicle $k$ can idle in period $t$. A penalty $\Theta$ is applied on the difference between the minimum and maximum vehicle workload, the latter represented by the total duration of all tours a vehicle executes over the planning horizon. Thus, the penalty serves as an incentive to balance workload among the vehicles.

We distinguish a tour from a trip, the latter being a sequence $\mathscr{S}$ of demand points visited by vehicle $k$ between two supply point visits. The supply point visits delimiting the trips may be in the same or in different periods. In a given solution, the set of supply point delimited trips performed by vehicle $k$ is denoted by $\mathfrak{S}_{k}$. Demand stochasticity affects trips through the probability of route failure, which is the probability of the total demand in trip $\mathscr{S} \in \mathfrak{S}_{k}$ exceeding the vehicle capacity $\Omega_{k}$. The recourse action is a visit to a supply point. The cost of this recourse action is $C_{\mathscr{S}}$, which is the average routing cost of going from the demand points in $\mathscr{S}$ to their nearest supply point and back. To control its degree of conservatism, this cost can be pre-multiplied by a Route Failure Cost Multiplier (RFCM) of $\psi$. We can also limit the probability of route failure to a maximum allowable level $\gamma^{\mathrm{RF}}$. The probability of route failure is further discussed in Section 4.3. All sets and parameters discussed above are summarized in Table 1 .

\section{Capturing Demand Stochasticity}

Our framework considers stochastic demands with all other parameters being fully deterministic. Below, we describe in detail how the unified framework captures stochastic demands. In particular, Section 4.1 outlines the forecasting of future demands and the minimum amount of forecasting information that the framework needs. Then, Sections 4.2 and 4.3 derive the probabilities of stock-out and route failure, respectively. We focus specifically on the issue of tractability and the fact that all probability information can be pre-computed or at least partially pre-processed.

\subsection{Demand Decomposition and Forecasting}

Given a demand point $i \in \mathcal{P}$ and a period $t \in \mathcal{T}$, the stochastic demand $\rho_{i t}$ decomposes as:

$$
\rho_{i t}=\mathbb{E}\left(\rho_{i t}\right)+\varepsilon_{i t}
$$

where $\mathbb{E}\left(\rho_{i t}\right)$ is the expected demand and $\varepsilon_{i t}$ is the error component. Let us represent $\varepsilon_{i t}, \forall t \in \mathcal{T}, i \in \mathcal{P}$ in the form of a vector as follows:

$$
\varepsilon=\left(\varepsilon_{11}, \ldots, \varepsilon_{1|\mathcal{T}|}, \varepsilon_{21}, \ldots, \varepsilon_{|\mathcal{P}||\mathcal{T}|}\right)
$$

The associated joint distribution is $\Phi$, and $\varepsilon \sim \Phi$ satisfies $\operatorname{var}(\varepsilon)=\mathrm{K}$, with $\mathrm{K}$ representing any covariance structure.

Definition 1. A forecasting model provides the expected demands $\mathbb{E}\left(\rho_{i t}\right), \forall t \in \mathcal{T}, i \in \mathcal{P}$ and the distribution $\Phi$ of $\varepsilon$.

Any forecasting model that complies with Definition 1 can be used. Moreover, the distribution $\Phi$ need not be theoretical. The only requirement is that we should be able to simulate it. Therefore, an empirical distribution is also admissible as we can sample from it. The forecasting model thus remains as general as possible, giving freedom for the use of methodologies suitable to the specific application area.

\subsection{Demand Point Probabilities}

Extending the terminology introduced in Section 3. we distinguish between a regular and an emergency delivery to demand point $i \in \mathcal{P}$. Let the binary decision variable $y_{i k t}=1$ denote a visit to demand point 
$i$ by vehicle $k \in \mathcal{K}$ in period $t \in \mathcal{T}$, and let $y_{i k t}=0$ denote otherwise. In other words, a regular delivery to demand point $i$ in period $t$ is one for which $y_{i k t}=1$ for some vehicle $k \in \mathcal{K}$. Contrarily, an emergency delivery is a recourse action performed in a state of stock-out in the absence of a regular delivery, i.e. for $t \in \mathcal{T}$ where $\sigma_{i t}=1$ and $y_{i k t}=0, \forall k \in \mathcal{K}$. Moreover, an emergency delivery always brings the inventory level at demand point $i$ to its capacity $\omega_{i}$. That is, for emergency deliveries we restrict the inventory policy to OU. This is in view of preserving tractability and is discussed in further detail below.

Definition 2. The initial inventory $I_{i 0}$ for each demand point $i \in \mathcal{P}$ is observed and known with certainty. It can be positive, zero or negative.

As a consequence, the probability of stock-out of demand point $i$ in period $t=0$ is either 0 or 1 . The inventory $I_{i t}$ in periods $t>0$ is affected by deliveries and demand realizations in previous periods.

Definition 3. In each period $t \in \mathcal{T}$, deliveries take place before demand realizations.

Let the variable $\Lambda_{i g}$ denote the inventory after a regular delivery to demand point $i$ in period $g$. As described in Section 3. $\Lambda_{i g} \in \mathcal{L}_{i}$. If there is no delivery to point $i$ during the planning horizon, $\Lambda_{i 0}=I_{i 0}$. Given that both the stock-out cost $\chi_{i}$ and the emergency delivery cost $\zeta_{i}$ for demand point $i$ are only paid in a state of stock-out, we are interested in calculating the probability of stock-out for all $i \in \mathcal{P}$ over the planning horizon. To do this, we extend the ideas presented in Markov et al. (2016a) in the context of the waste collection IRP. Consider a regular delivery to demand point $i$ in period $g \in \mathcal{T}$. We identify four possible ways of reaching a state of stock-out. Given the stochastic demand decomposition formula (1) and the action sequence in Definition 3 , their probabilities are formulated as:

- Reaching a state of stock-out in period $g+1$ from a regular delivery in period $g$. Its probability is unconditional and is given by:

$$
\mathbb{P}\left(\Lambda_{i g}-\rho_{i g} \leqslant 0\right)=\mathbb{P}\left(\varepsilon_{i g} \geqslant \Lambda_{i g}-\mathbb{E}\left(\rho_{i g}\right)\right) .
$$

- Reaching a state of stock-out in periods later than $g+1$ from a regular delivery in period $g$. Its probability is conditional and is given by:

$$
\begin{gathered}
\mathbb{P}\left(\Lambda_{i g}-\sum_{t=g}^{h} \rho_{i t} \leqslant 0 \mid \Lambda_{i g}-\sum_{t=g}^{h-1} \rho_{i t}>0\right)= \\
=\mathbb{P}\left(\sum_{t=g}^{h} \varepsilon_{i t} \geqslant \Lambda_{i g}-\sum_{t=g}^{h} \mathbb{E}\left(\rho_{i t}\right) \mid \sum_{t=g}^{h-1} \varepsilon_{i t}<\Lambda_{i g}-\sum_{t=g}^{h-1} \mathbb{E}\left(\rho_{i t}\right)\right), \quad \forall h>g .
\end{gathered}
$$

- Reaching a state of stock-out in period $g^{\prime}+1$ from a state of stock-out in period $g^{\prime}>g$. Its probability is unconditional and is calculated as a special case of formula (3) as follows:

$$
\mathbb{P}\left(\omega_{i}-\rho_{i g^{\prime}} \leqslant 0\right)=\mathbb{P}\left(\varepsilon_{i g^{\prime}} \geqslant \omega_{i}-\mathbb{E}\left(\rho_{i g^{\prime}}\right)\right), \quad \forall g^{\prime}>g .
$$

- Reaching a state of stock-out in periods later than $g^{\prime}+1$ from a state of stock-out in period $g^{\prime}>g$. Its probability is conditional and is calculated as a special case of formula (4) as follows:

$$
\begin{gathered}
\mathbb{P}\left(\omega_{i}-\sum_{t=g^{\prime}}^{h} \rho_{i t} \leqslant 0 \mid \omega_{i}-\sum_{t=g^{\prime}}^{h-1} \rho_{i t}>0\right)= \\
=\mathbb{P}\left(\sum_{t=g^{\prime}}^{h} \varepsilon_{i t} \geqslant \omega_{i}-\sum_{t=g^{\prime}}^{h} \mathbb{E}\left(\rho_{i t}\right) \mid \sum_{t=g^{\prime}}^{h-1} \varepsilon_{i t}<\omega_{i}-\sum_{t=g^{\prime}}^{h-1} \mathbb{E}\left(\rho_{i t}\right)\right), \quad \forall h>g^{\prime}>g .
\end{gathered}
$$

Appendix A proves that the calculation of the probabilities of overflow for a collection problem is identical. For a demand point $i$ with a regular delivery in period $g$, the above probabilities are mapped on a binary 
tree as illustrated in Figure1, in which the state of stock-out is shaded in gray. The probability of stock-out in period $t>g$ is the sum of the probabilities of all possible paths reaching a state $\sigma_{i t}=1$ starting from the root node with an inventory after delivery of $\Lambda_{i g}$ in period $g$. The probability of stock-out in period $g$ is calculated on the basis of the previous tree, and it is 0 or 1 when $g=0$. Thus, we arrive at the general expression for the probability of stock-out of demand point $i$ in period $t$ :

$$
\mathrm{p}_{i t}^{\text {DP }}=\mathbb{P}\left(\sigma_{i t}=1 \mid \Lambda_{i m}: m=\max \left(0, g \in \mathcal{T}: g<t: \exists k \in \mathcal{K}: y_{i k g}=1\right)\right) .
$$

It correctly defines the probability of stock-out in period $t$ as conditional on the inventory after delivery $\Lambda_{i m}$ set in the most recent regular delivery, identified for each demand point $i$ by the index $m$. The max operator returns the period 0 if the demand point has not had any regular deliveries prior to period $t$. In other words, expression 7 indicates that once the routing and delivery decisions in period $m$ have been made, stock-out probabilities in each subsequent period are calculated on the basis of the tree presented in Figure 1 using probability formulas 3 to 6 .

Proposition 1. Under a discretized ML policy, the stock-out probabilities in expression (7) can be precomputed. Moreover, the number of probabilities to pre-compute grows linearly with the number of discrete levels.

Proof. For the unconditional probabilities (3) and (5), the number of distinct expressions to evaluate is linear in the number of periods $t \in \mathcal{T}$, while for the conditional probabilities (4) and (6) it is polynomial. As a consequence, the resulting stock-out probabilities in formula (7) can be efficiently pre-computed. Secondly, the formula defines the probability of $\sigma_{i t}=1$ as conditional only on the inventory level $\Lambda_{i m}$ chosen in the most recent delivery period $m$. The probabilities (7) for demand point $i$ are precomputed for each $r \in \mathcal{L}_{i}$, hence the number probabilities to pre-compute grows linearly with the number of discrete levels.

Figure 1: Demand Point State Probability Tree

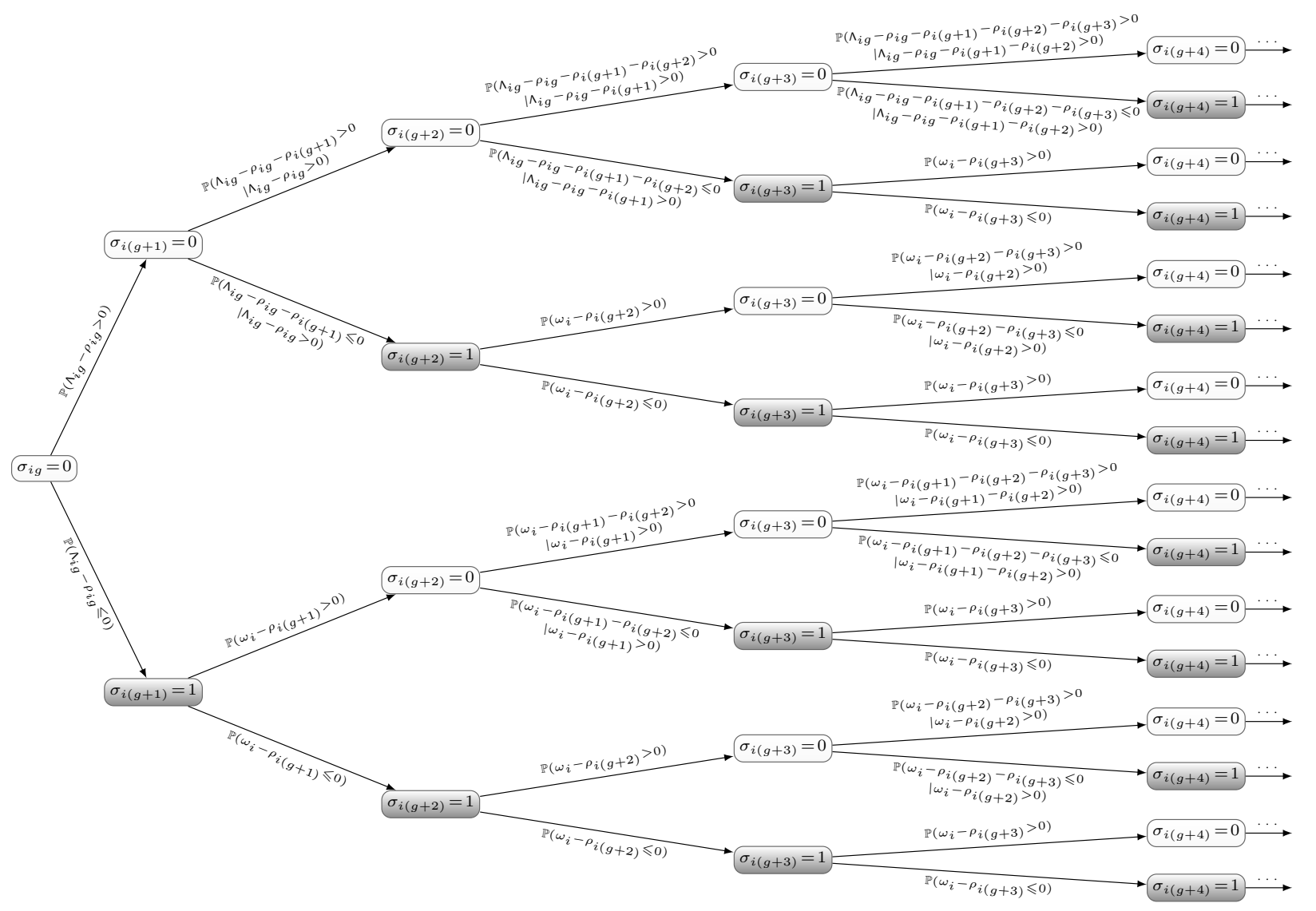


The emergency deliveries still apply an OU policy, otherwise the combinatorial dimension would become intractable. Appendix B demonstrates the use of simulation to pre-compute the stock-out probabilities (3)(6) given a general distribution $\Phi$ and a covariance structure $\mathrm{K}$ among the error terms $\varepsilon$ in formula (2). In Section 3 it was mentioned that the discretized ML inventory policy is used for the sake of tractability in order to avoid cumbersome calculations at runtime. Indeed, as shown in the proof to Proposition 1, the ability to pre-compute the stock-out probabilities relies on the discrete values of $\Lambda_{i m}$.

Finally, for expression (7) to be rigorously defined, the chosen inventory after delivery $\Lambda_{i m}$ must be feasible. This condition always holds for the OU policy, which delivers up to capacity. However, the ML policy implies a non-negative probability of the chosen $\Lambda_{i m}$ being lower than the realized inventory. There are several possibilities for handling this, including:

- Performing no delivery. This approach leads to an additional layer of conditionality in the calculation of the stock-out probabilities, given that there may be no actual delivery in the most recent visit period $m$ in formula (7). Even if the stock-out probabilities can still be pre-computed, there is a marked increase in complexity which makes the approach unattractive.

- Picking up the excess inventory. This approach destroys the monotonically decreasing property of the residual quantity on the vehicle at each successive demand point. The route failure probabilities now become conditional on the previous demand point visits. As described in Section 4.3 below, route failure probabilities are calculated at runtime. Thus, additional complications in the probability expression would lead to tractability issues.

- Discarding the excess inventory. This approach is the most appealing from a modeling point of view as it allows the use of the chosen values $\Lambda_{i m}$ both in the calculation of stock-out and route failure probabilities. Discarding excess inventory can in principle be penalized, its probability being a straightforward extension of formula (7). Hence, we formulate the following assumption.

Assumption 1. A regular delivery to demand point $i \in \mathcal{P}$ in period $t \in \mathcal{T}$ discards any inventory above the chosen level $\Lambda_{i t}$.

Assumption 1 underlies the calculation of the stock-out probabilities as defined by formula (7) as well as the calculation of the route failure probabilities discussed in Section 4.3 next.

\subsection{Route Failure Probabilities}

Recalling the notation introduced in Section 3 , for each vehicle $k$ in a given solution, we identify the set of supply point delimited trips $\mathfrak{S}_{k}$. Let the binary decision variables $x_{i j k t}=1$ if vehicle $k$ traverses arc $(i, j)$ in period $t$, and 0 otherwise. For a vehicle $k$, given $x_{i j k t}, \forall i, j \in \mathcal{P}, t \in \mathcal{T}$, Algorithm 1 builds the set of supply point delimited trips $\mathfrak{S}_{k}$, where as before $\mathscr{S}$ is a trip in $\mathfrak{S}_{k}$. The algorithm identifies the sequence of visits using the routing variables $x_{i j k t}$ for each period $t \in \mathcal{T}$. A visit to a supply point starts a new trip $\mathscr{S}$. In the context of multi-period trips, the supply points delimiting the trips $\mathscr{S} \in \mathfrak{S}_{k}$ may be visited in different periods $t$. Thus, each trip $\mathscr{S}$ is further decomposed into sets $\mathcal{S}_{t}$, where $\mathcal{S}_{t} \in \mathscr{S}$ is the set of demand points in trip $\mathscr{S}$ that are visited in period $t$.

The above notation is used in the formulation of the probability of route failure, which is the probability of the total demand in trip $\mathscr{S} \in \mathfrak{S}_{k}$ exceeding the vehicle capacity $\Omega_{k}$. We define the quantity $\Gamma_{\mathscr{S}}$ delivered in trip $\mathscr{S}$ as:

$$
\begin{gathered}
\Gamma_{\mathscr{S}}=\sum_{\mathcal{S}_{0} \in \mathscr{S}} \sum_{s \in \mathcal{S}_{0}}\left(\Lambda_{s 0}-I_{s 0}\right)+\sum_{t \in \mathcal{T} \backslash 0} \sum_{\mathcal{S}_{t} \in \mathscr{S}} \sum_{s \in \mathcal{S}_{t}}\left(\Lambda_{s t}-\Lambda_{s m}+\sum_{h=m}^{t-1} \rho_{s h}\right), \\
\text { where } m=\max \left(0, g \in \mathcal{T}: g<t: \exists k^{\prime} \in \mathcal{K}: y_{s k^{\prime} g}=1\right) .
\end{gathered}
$$

The first summand in formula (8) represents the quantity delivered in period $t=0$, for which there is no uncertainty, while the second summand defines the quantity delivered in periods $t>0$ given the action sequence in Definition 3 and the inventory after delivery under Assumption 11. Similar to formula (7), the 


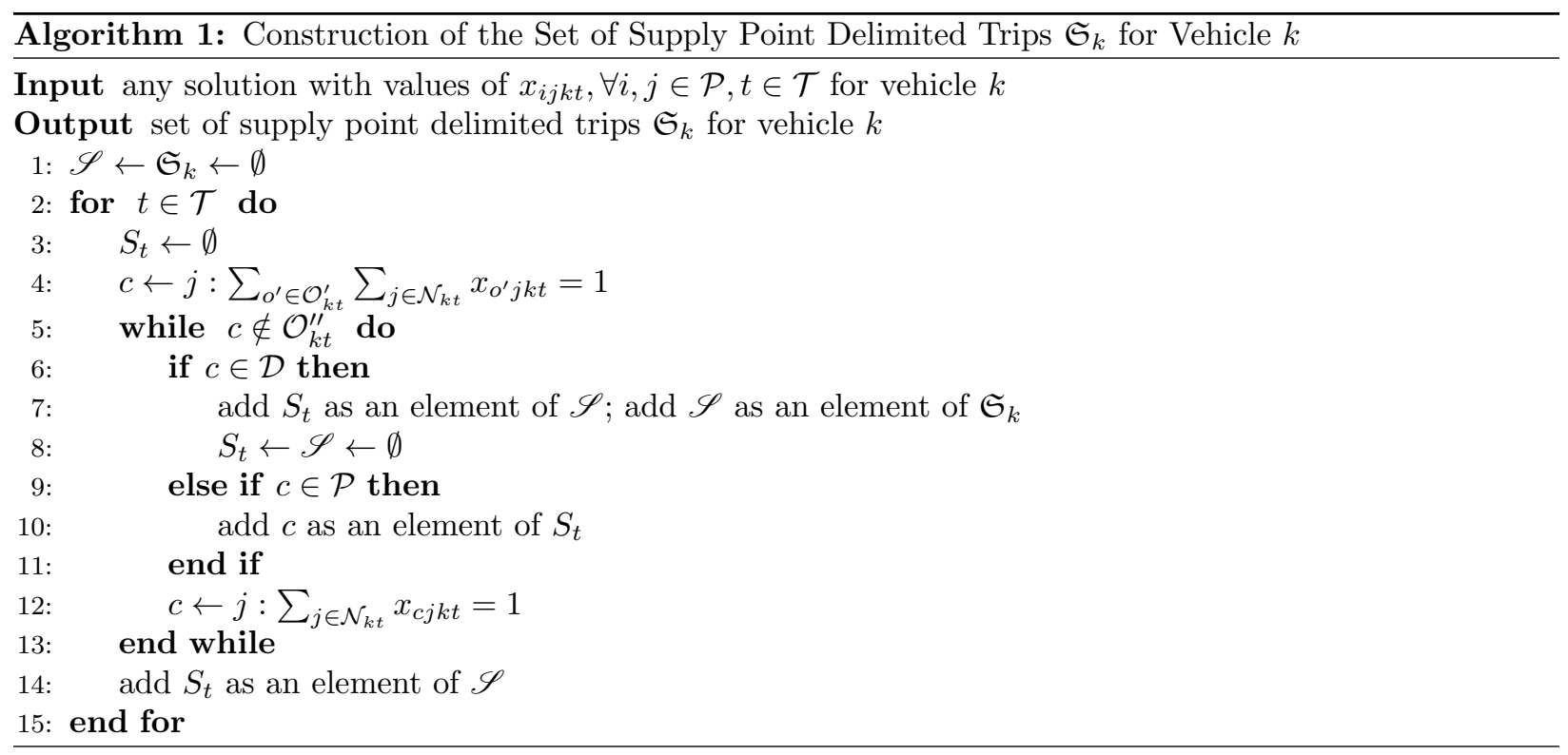

index $m$ identifies the most recent regular delivery to point $s$. Having defined $\Gamma_{\mathscr{S}}$, the probability of route failure in trip $\mathscr{S} \in \mathfrak{S}_{k}$ performed by vehicle $k \in \mathcal{K}$ becomes:

$$
\mathrm{p}_{\mathscr{S}, k}^{\mathrm{RF}}=\mathbb{P}\left(\Gamma_{\mathscr{S}}>\Omega_{k}\right) .
$$

Formula (9) captures the probability of multiple route failures in each trip $\mathscr{S}$. Unlike in the case of the stock-out probabilities, the probabilities of route failure depend on the optimization decisions, in particular the sets $\mathfrak{S}_{k}, \forall k \in \mathcal{K}$ at each solution and the values of $\Lambda_{s t}$ and $\Lambda_{s m}$. As a consequence, these probabilities cannot be precomputed. Moreover, the distribution of $\Gamma_{\mathscr{S}}$ is unknown except for special cases, e.g. when the distributions $\Phi$ of $\varepsilon$ is the normal. This motivates the following assumption.

Assumption 2. The calculation of the route failure probabilities assumes independent and identically distributed (iid) error terms $\varepsilon_{i t}$ drawn from any distribution $\Phi$ of $\varepsilon$. Consider $\varepsilon$ as defined by equation (2) above. We impose the iid assumption on the error terms by stipulating:

$$
\Phi(\varepsilon)=\prod_{t \in \mathcal{T}} \prod_{i \in \mathcal{P}} \Phi^{\prime}\left(\varepsilon_{i t}\right),
$$

where $\Phi^{\prime}$ is the marginal cumulative distribution function of $\varepsilon_{i t}$.

Assumption 2 is widely used in the routing literature in the context of normally distributed demands (Gendreau et al., 2016), and may cause the true effect to be under- or overestimated. In our framework, it is only imposed for the calculation of the route failure probabilities, without imposing normality or other distributional restrictions. In essence, the assumption renders the demands $\rho_{s h}$ in formula (8) independent of the particular $s \in \mathcal{P}$ and $h \in \mathcal{T}$. With Assumption 2 the distribution of $\Gamma_{\mathscr{S}}$ depends only on the number $n$ of summed demands, where $n$ is bounded above by $|\mathcal{P}|(|\mathcal{T}|-1)$. Since Definition 3 stipulates that in each period deliveries are performed before demand realizations, the demands of the last period of the planning horizon cannot be served during the planning horizon. Hence the bound, which considers a trip serving all demands realized before the last period of the planning horizon. Given this bound, an empirical distribution function can be derived for each $n$ and used at runtime.

The use of simulation for this partial pre-processing of the route failure probabilities through the derivation of empirical distribution functions is elaborated in Appendix C. In addition, the numerical experiments in Section 7.2 demonstrate that the use of these pre-processed distributions at runtime has an insignificant effect on the computational burden. Finally, the calculation of both the stock-out and the route failure probability depends on the structural assumption below. 
Assumption 3. Supply points have unlimited inventories.

In many practical applications, for example waste collection, supply point inventories are irrelevant. From a more fundamental point of view, this modeling choice is also due to the complex propagations of uncertainty that tracking supply point inventories would entail. For example, it is unclear, unless explicitly postulated as a rule, which supply points would be affected by the stock-out and route failure recourse actions described above and by how much. Another example is related to the residual quantity still on the vehicle when reaching a supply point. That is, if the demands of the previously visited demand points were lower than expected, the vehicle would need to load less than expected at the supply point due to the residual quantity already on board. This directly affects tracking the supply point inventory. Given the possibility of multiple supply point visits in each period, correctly accounting for this uncertainty propagation leads to complex conditionality which is nearly impossible to evaluate without expensive simulation runs.

\section{Optimization Model}

This section develops the objective function and the constraints of the optimization model. The formulation is presented and interpreted from a distribution point of view. Nevertheless, since collection can be viewed as the distribution of empty space, the optimization model itself does not change. To complete the notation, we provide the list of decision variables, including those already used in Section 4 . Starting with the binary variables, $x_{i j k t}=1$ if vehicle $k$ traverses arc $(i, j)$ in period $t, 0$ otherwise; $y_{i k t}=1$ if point $i \in \mathcal{O} \cup \mathcal{P} \cup \mathcal{D}$ is visited by vehicle $k$ in period $t, 0$ otherwise; $z_{k t}=1$ if vehicle $k$ is used in period $t, 0$ otherwise; $c_{i r}=1$ if visit day combination $r \in \mathcal{C}_{i}$ is assigned to demand point $i, 0$ otherwise; $\ell_{\text {irt }}=1$ if inventory level $r \in \mathcal{L}_{i}$ is chosen for demand point $i$ in period $t, 0$ otherwise. Moving to the continuous variables, $q_{i k t}$ is the expected delivery quantity to demand point $i$ by vehicle $k$ in period $t ; Q_{i k t}$ is the expected cumulative quantity delivered by vehicle $k$ arriving at point $i \in \mathcal{O} \cup \mathcal{P} \cup \mathcal{D}$ in period $t ; I_{i t}$ is the expected inventory at demand point $i$ at the start of period $t$, i.e. before delivery; $S_{i k t}$ is the start-of-service time of vehicle $k$ at point $i \in \mathcal{O} \cup \mathcal{P} \cup \mathcal{D}$ in period $t ; \underline{b}_{k t}$ and $\bar{b}_{k t}$ are the lower and upper bound on the tour duration of vehicle $k$ in period $t$; and $\underline{B}$ and $\bar{B}$ are the lower and upper bound on the workload for each vehicle. These definitions also appear in Table 1

\subsection{Objective Function}

The objective function consists of four deterministic and two stochastic components, all of which are independent of one another. Different combinations of these make it possible to model a variety of routing problems, whether with deterministic or stochastic demands. Starting with the deterministic components, the Expected Inventory Holding Cost (EIHC) is the cost due to keeping the expected inventory at the demand points. Since the inventories in the first period after the end of the planning horizon are completely determined by the decisions taken during the planning horizon, the EIHC is computed for $t \in \mathcal{T} \cup \mathcal{T}^{+}$, where $\mathcal{T}^{+}$is the planning horizon shifted right by one period. The EIHC is formulated as:

$$
\mathrm{EIHC}=\sum_{t \in \mathcal{T} \cup \mathcal{T}+} \sum_{i \in \mathcal{P}} \eta_{i} I_{i t}
$$

The Visit Cost (VC) component applies a cost for each visit to a demand point:

$$
\mathrm{VC}=\sum_{t \in \mathcal{T}} \sum_{k \in \mathcal{K}} \sum_{i \in \mathcal{P}} \xi_{i} y_{i k t}
$$

The Routing Cost (RC) component applies the three vehicle-related costs, namely the per-period deployment $\operatorname{cost} \varphi_{k}$, the unit-distance running $\operatorname{cost} \beta_{k}$ and the unit-time running cost $\theta_{k}$, for each period $t \in \mathcal{T}$ and each vehicle $k \in \mathcal{K}$ :

$$
\mathrm{RC}=\sum_{t \in \mathcal{T}} \sum_{k \in \mathcal{K}}\left(\varphi_{k} z_{k t}+\beta_{k} \sum_{i \in \mathcal{N}_{k t}} \sum_{j \in \mathcal{N}_{k t}} \pi_{i j} x_{i j k t}+\theta_{k}\left(\sum_{o^{\prime \prime} \in \mathcal{O}_{k t}^{\prime \prime}} S_{o^{\prime \prime} k t}-\sum_{o^{\prime} \in \mathcal{O}_{k t}^{\prime}} S_{o^{\prime} k t}\right)\right) .
$$


The Workload Balancing (WB) component attempts to balance the workload over the planning horizon equally among the vehicles by penalizing the difference between the lowest and the highest vehicle workload:

$$
\mathrm{WB}=\Theta(\bar{B}-\underline{B}) .
$$

Moving to the stochastic components, the Expected Stock-Out and Emergency Delivery Cost (ESOEDC) component, as its name suggests, reflects the stock-out and emergency delivery cost and writes as:

$$
\mathrm{ESOEDC}=\sum_{t \in \mathcal{T} \cup \mathcal{T}^{+}} \sum_{i \in \mathcal{P}}\left(\chi_{i}+\zeta_{i}-\zeta_{i} \sum_{k \in \mathcal{K}} y_{i k t}\right) \mathrm{p}_{i t}^{\mathrm{DP}},
$$

where the probability of stock-out at the demand point $\mathbf{p}_{i t}^{\text {DP }}$ is defined by formula (7). For demand point $i$ in period $t$, the ESOEDC component applies the stock-out cost $\chi_{i}$ and the emergency delivery cost $\zeta_{i}$ in case there is no regular delivery in that period, and only the stock-out cost $\chi_{i}$ in case there is a regular delivery. Although there is no uncertainty in period $t=0$, we still need to pay the stock-out cost if the demand point is in a state of stock-out. Since the stock-out probabilities in the first period after the end of the planning horizon are completely determined by the decisions taken during the planning horizon, the ESOEDC is also computed for $t \in \mathcal{T} \cup \mathcal{T}^{+}$.

The Expected Route Failure Cost (ERFC) captures the risk of the vehicles running out of capacity before reaching the next scheduled visit to a supply point due to higher than expected demands. It is expressed as:

$$
\mathrm{ERFC}=\sum_{k \in \mathcal{K}} \sum_{\mathscr{S} \in \mathfrak{S}_{k}} \psi C_{\mathscr{S}} \mathrm{p}_{\mathscr{S}, k}^{\mathrm{RF}}
$$

where the probability of route failure $\mathrm{p}_{\mathscr{S}, k}^{\mathrm{RF}}$ is defined by formula $(9)$. As in Section $4.3 \mathfrak{S}_{k}$ is the set of supply point delimited trips executed by vehicle $k, \mathscr{S} \in \mathfrak{S}_{k}$ is a particular trip in that set, and $C_{\mathscr{S}}$ is the average routing cost of going from the demand points in $\mathscr{S}$ to the nearest supply point and back. The parameter $\psi \in[0,1]$, which we refer to as the Route Failure Cost Multiplier (RFCM), is used to scale up or down the degree of conservatism of the ERFC component.

The resulting objective is non-linear due to the non-linear nature of the ESOEDC and ERFC components. In the former, the degree of $y_{i k t}$ is higher than one due to the implicit presence of $y_{i k t}$ also in $\mathrm{p}_{i t}^{\text {DP }}$ as defined by formula $(7)$. In the latter, the probability $\mathrm{p}_{\mathscr{S}, k}^{\mathrm{RF}}$ is in general non-linear in the value of $\Gamma_{\mathscr{S}}$ as given by the relationship in formula (9). The objective function $z$ is formulated as:

$$
\min z=\mathrm{EIHC}+\mathrm{VC}+\mathrm{RC}+\mathrm{WB}+\mathrm{ESOEDC}+\mathrm{ERFC},
$$

where the RC, ESOEDC and ERFC components are generalized from Markov et al. (2016a).

The objective function formulation (17) implies here-and-now decisions, integrating the probabilities of stockouts and route failures over the planning horizon $\mathcal{T}$. On the other hand, demands are typically revealed dynamically over $\mathcal{T}$. To adapt to this dynamic nature, the problem can be solved in a period-based rolling horizon fashion, only implementing the decisions for period $t=0$ for each roll-over. In effect, the probabilistic information regarding stock-outs and route failures, as well as their approximate costs, helps make betterinformed decisions about routing and delivery quantities for $t=0$. This probabilistic information is updated with new forecasts at each roll-over.

\subsubsection{Overestimation of the Real Cost.}

To keep the approach tractable, the objective function is a simplification of the real cost and as a result it deviates from it. More precisely, the modeling simplifications are summarized as follows.

Assumption 4. All terms of the objective function (17), except the ESOEDC, ignore the cost effect of demand points stocking out earlier than expected. 
Fully capturing this effect would imply developing complex probability expressions for all cost components. Alternatively, the probability calculations can be simplified through the imposition of other restrictive assumptions. A case in point is the work of Trudeau and Dror (1992), who impose a maximum of one delivery and stock-out for each demand point over the planning horizon. Given that there is an emergency delivery recourse action, such points are skipped in subsequent tours. This makes it easier to formulate analytical expressions that capture the above effect. Our framework does not limit the number of deliveries and stockouts over the planning horizon. In this regard, Trudeau and Dror s (1992) approach of skipping demand points stocking out earlier than expected is only one of a range of possible reaction policies.

Definition 4. A reaction policy is a response to the recourse action by changes later in the planning horizon.

Reaction policies can vary from doing nothing to completely re-optimizing the subsequent decisions. The possibility of multiple stock-outs followed by emergency deliveries leads to the conditional dependence of reaction policies, with consequences on tractability. In particular, it precludes the partial pre-processing of the route failure probabilities discussed in Section 4.3 .

Proposition 2. In the absence of the EIHC component, objective function (17) overestimates the real cost.

Proof. Consider demand point $i \in \mathcal{P}$ that stocks out in period $g$ and is not visited for a regular delivery in period $g$. For a do-nothing reaction policy, there is no effect on the $\mathrm{VC}, \mathrm{RC}$ and $\mathrm{WB}$ components as it implies no change in the routing decisions. The ESOEDC component already captures the probability of demand points stocking out earlier than expected. For the effect on the ERFC component, we identify two cases:

1. There is a vehicle $k \in \mathcal{K}$ that visits point $i$ for a regular delivery in trip $\mathscr{S} \in \mathfrak{S}_{k}$ in period $t>g$. Given the emergency delivery to point $i$ in period $g$, vehicle $k$ will deliver less than expected in trip $\mathscr{S}$, reducing the probability of route failure $\mathrm{p}_{\mathscr{S}, k}^{\mathrm{RF}}$ according to formula $(9)$.

2. Alternatively, there is no trip $\mathscr{S}$ that visits point $i$ in period $t>g$. Therefore, $\mathrm{p}_{\mathscr{S}, k}^{\mathrm{RF}}$ remains unaffected for all trips $\mathscr{S} \in \mathfrak{S}_{k}, \forall k \in \mathcal{K}$.

Given the existence of a more sophisticated reaction policy, the overestimation of the real cost may be higher. The above discussion considers no EIHC component. In a distribution problem, a stock-out in period $g$, followed by an emergency delivery, results in inventory levels $I_{i t}$ being higher than expected for $t>g$. Thus, expression (11) of the EIHC underestimates the inventory holding cost for $t>g$. It is the contrary for a collection problem, where an overflow in period $g$, followed by an emergency collection, results in inventory levels $I_{i t}$ being lower than expected for $t>g$. In this case, expression (11) of the EIHC overestimates the inventory holding cost.

The overestimation due to the do-nothing reaction policy is straightforward to evaluate through simulation on the final solution. On the other hand, the evaluation of the effect of an optimal reaction policy would require re-optimizing the decisions after each stock-out. Section 7.2 .2 presents a trivial upper bound on the optimal reaction policy for a case study based on a waste collection IRP and shows that the practical effect of Assumption 4 is marginal.

\subsection{Deterministic Constraints}

The deterministic constraints use expected values of the demand-related decision variables, the stochasticity having been incorporated in the objective function. This is a policy-based approach capturing the intention of the decision maker to avoid stock-outs and route failures in the expected sense. The alternative, with the use of probabilistic constraints, is presented in Section 5.3 next.

Starting with the basic routing constraints, tours must have an origin and a destination depot, as ensured by constraints (18), which also allow for simple relocation tours not visiting any demand or supply points. Constraints (19) and 20) forbid returns to the origin depots and departures from the destination depots. Given the possibility of open tours, we need to ensure that a vehicle's destination depot in period $t$ is the same as its origin depot in period $t+1$. Constraints (21) propagate this condition through the planning 
horizon. Further on, constraints (22) and (23) link the visit and the routing variables, and constraints (24) ensure that a demand point is visited at most once per period. Accessibility restrictions and continuity of service are enforced by constraints 25 . Constraints 26 ensure flow conservation.

$$
\begin{array}{ll}
\sum_{o^{\prime} \in \mathcal{O}_{k t}^{\prime}} \sum_{j \in \mathcal{N}_{k t}} x_{o^{\prime} j k t}=\sum_{i \in \mathcal{N}_{k t}} \sum_{o^{\prime \prime} \in \mathcal{O}_{k t}^{\prime \prime}} x_{i o^{\prime \prime} k t}, & \forall t \in \mathcal{T}, k \in \mathcal{K} \\
\sum_{i \in \mathcal{N}_{k t}} x_{i o^{\prime} k t}=0, & \forall t \in \mathcal{T}, k \in \mathcal{K}, o^{\prime} \in \mathcal{O}_{k t}^{\prime \prime} \\
\sum_{j \in \mathcal{N}_{k t}} x_{o^{\prime \prime} j k t}=0, & \forall t \in \mathcal{T}, k \in \mathcal{K}, o^{\prime \prime} \in \mathcal{O}_{k t}^{\prime \prime} \\
\sum_{i \in \mathcal{N}_{k t}} x_{i o k t}=\sum_{j \in \mathcal{N}_{k(t+1)}} x_{o j k(t+1)}, & \forall t \in \mathcal{T}, k \in \mathcal{K}, o \in \mathcal{O}_{k t}^{\prime \prime} \cap \mathcal{O}_{k(t+1)}^{\prime} \\
y_{i k t}=\sum_{j \in \mathcal{N}_{k t}} x_{i j k t}, & \forall t \in \mathcal{T}, k \in \mathcal{K}, i \in \mathcal{N}_{k t} \backslash \mathcal{O}_{k t}^{\prime \prime} \\
y_{j k t}=\sum_{i \in \mathcal{N}_{k t}} x_{i j k t}, & \forall t \in \mathcal{T}, k \in \mathcal{K}, j \in \mathcal{O}_{k t}^{\prime \prime} \\
\sum_{k \in \mathcal{K}} y_{i k t} \leqslant 1, & \forall t \in \mathcal{T}, i \in \mathcal{P} \\
y_{i k t} \leqslant \alpha_{i k t}, & \forall t \in \mathcal{T}, k \in \mathcal{K}, i \in \mathcal{P} \cup \mathcal{D} \\
\sum_{i \in \mathcal{N}_{k t}} x_{i j k t}=\sum_{i \in \mathcal{N}_{k t}} x_{j i k t}, & \forall t \in \mathcal{T}, k \in \mathcal{K}, j \in \mathcal{P} \cup \mathcal{D}
\end{array}
$$

The periodicity aspect is established by constraints (27), which assign exactly one visit period combination to each demand point, and constraints (28), which in turn limit visits to the periods corresponding to the assigned visit period combination (Cordeau et al. 1997). The set $\mathcal{C}_{i}$ may contain visit period combinations with different frequencies, which makes the visit frequency part of the optimization decisions.

$$
\begin{array}{ll}
\sum_{r \in \mathcal{C}_{i}} c_{i r}=1, & \forall i \in \mathcal{P} \\
\sum_{k \in \mathcal{K}} y_{i k t}-\sum_{r \in \mathcal{C}_{i}} \alpha_{r t} c_{i r}=0, & \forall t \in \mathcal{T}, i \in \mathcal{P}
\end{array}
$$

The inventory constraints at the demand points comply with the action sequence in Definition 3 Constraints 299 track the expected inventory in period $t$ as a function of the expected inventory, the quantity delivered to the point, and its expected demand in period $t-1$. Constraints 30 ensure that the expected inventory remains non-negative, and constraints (31) force a delivery if the inventory is below zero in period $t=0$. Constraints (32)-(35) define the choice of a discrete inventory level and the delivery quantity it entails. In particular, constraints (32) stipulate that if a demand point is visited, then a discrete inventory level after delivery must be chosen. Constraints (33) and (34) provide a lower and an upper bound on the delivery quantity which, if the point is visited, is equal to the difference between the chosen discrete inventory level after delivery and the expected inventory. The latter also imply that if the point is visited, the chosen level will be higher than the expected inventory. Constraints 35 force the delivery quantity to zero if the point is not visited. The big- $M$ values in constraints (33) and (35) are equal to $2 \omega_{i}$ for $t=0$ and to $\omega_{i}$ otherwise, reflecting the fact that the expected delivery quantity cannot exceed demand point capacity, except in period $t=0$.

$$
\begin{array}{ll}
I_{i t}=I_{i(t-1)}+\sum_{k \in \mathcal{K}} q_{i k(t-1)}-\mathbb{E}\left(\rho_{i(t-1)}\right), & \forall t \in \mathcal{T}^{+}, i \in \mathcal{P} \\
I_{i t} \geqslant 0, & \forall t \in \mathcal{T}^{+}, i \in \mathcal{P} \\
-I_{i 0} \leqslant \omega_{i} \sum_{k \in \mathcal{K}} y_{i k 0}, & \forall i \in \mathcal{P}
\end{array}
$$




$$
\begin{array}{ll}
\sum_{k \in \mathcal{K}} y_{i k t}-\sum_{r \in \mathcal{L}_{i}} \ell_{i r t}=0, & \forall t \in \mathcal{T}, i \in \mathcal{P} \\
q_{i k t} \geqslant \sum_{r \in \mathcal{L}_{i}} r \ell_{i r t}-I_{i t}-M\left(1-y_{i k t}\right), & \forall t \in \mathcal{T}, k \in \mathcal{K}, i \in \mathcal{P} \\
q_{i k t} \leqslant \sum_{r \in \mathcal{L}_{i}} r \ell_{i r t}-I_{i t}+\omega_{i}\left(1-y_{i k t}\right), & \forall t \in \mathcal{T}, k \in \mathcal{K}, i \in \mathcal{P} \\
q_{i k t} \leqslant M y_{i k t}, & \forall t \in \mathcal{T}, k \in \mathcal{K}, i \in \mathcal{P}
\end{array}
$$

In the context of vehicle capacities, constraints (36) limit the cumulative quantity delivered by the vehicle at each demand point, while constraints (37) reset it to zero at the supply points. Keeping track of the cumulative quantity delivered by the vehicle is achieved by constraints (38). In the context of multi-period trips, constraints (39) link the quantity delivered by the vehicle from one period to the next. Forcing the vehicle to visit a supply point immediately after the origin depot or immediately before the destination depot applies to certain problems and is exemplified in Section 6 next.

$$
\begin{array}{ll}
q_{i k t} \leqslant Q_{i k t} \leqslant \Omega_{k}, & \forall t \in \mathcal{T}, k \in \mathcal{K}, i \in \mathcal{P} \\
Q_{i k t}=0, & \forall t \in \mathcal{T}, k \in \mathcal{K}, i \in \mathcal{D} \\
Q_{i k t}+q_{j k t} \leqslant Q_{j k t}+\Omega_{k}\left(1-x_{i j k t}\right), & \forall t \in \mathcal{T}, k \in \mathcal{K}, i \in \mathcal{N}_{k t}, j \in \mathcal{N}_{k t} \backslash \mathcal{D} \\
Q_{o^{\prime} k(t+1)} \geqslant Q_{o^{\prime \prime} k t}, & \forall t \in \mathcal{T}, k \in \mathcal{K}, o^{\prime} \in \mathcal{O}_{k t}^{\prime}, o^{\prime \prime} \in \mathcal{O}_{k t}^{\prime \prime}
\end{array}
$$

The next set of constraints expresses the intra-period temporal characteristics of the problem. Constraints (40) calculate the start-of-service time at each point and eliminate the possibility of subtours. Constraints (41) enforce the time windows. Constraints $(42$ bound the tour duration from above and below. Constraints 43 enforce the maximum tour duration, and with it availabilities and vehicle use. Constraints (44) and 45 bound the total tour duration over the planning horizon for each vehicle. The difference between $\underline{B}$ and $\bar{B}$ is the difference between the lowest and highest vehicle workload over the planning horizon, which is penalized by the WB component in the objective function.

$$
\begin{array}{ll}
S_{i k t}+\delta_{i}+\tau_{i j k t} \leqslant S_{j k t}+\left(\mu_{i}+\delta_{i}+\tau_{i j k t}\right)\left(1-x_{i j k t}\right), & \forall t \in \mathcal{T}, k \in \mathcal{K}, i \in \mathcal{N}_{k t}, j \in \mathcal{N}_{k t} \\
\lambda_{i} y_{i k t} \leqslant S_{i k t} \leqslant \mu_{i} y_{i k t}, & \forall t \in \mathcal{T}, k \in \mathcal{K}, i \in \mathcal{N}_{k t} \\
\underline{b}_{k t} \leqslant \sum_{0_{o^{\prime \prime} \in \mathcal{O}_{k t}^{\prime \prime}}} S_{o^{\prime \prime} k t}-\sum_{o^{\prime} \in \mathcal{O}_{k t}^{\prime}} S_{o^{\prime} k t} \leqslant \bar{b}_{k t}, & \forall t \in \mathcal{T}, k \in \mathcal{K} \\
\bar{b}_{k t} \leqslant \mathrm{H}_{k t} z_{k t}, & \forall t \in \mathcal{T}, k \in \mathcal{K} \\
\underline{B} \leqslant \sum_{t \in \mathcal{T}} \underline{b}_{k t}, & \forall k \in \mathcal{K} \\
\bar{B} \geqslant \sum_{t \in \mathcal{T}} \bar{b}_{k t}, & \forall k \in \mathcal{K}
\end{array}
$$

Finally, lines 46-47) establish the variable domains.

$$
\begin{array}{ll}
x_{i j k t}, y_{i k t}, z_{k t}, c_{i r^{\prime}}, \ell_{i r^{\prime \prime} t} \in\{0,1\}, & \forall t \in \mathcal{T}, k \in \mathcal{K}, i, j \in \mathcal{N}_{k t}, r^{\prime} \in \mathcal{C}_{i}, r^{\prime \prime} \in \mathcal{L}_{i} \\
q_{i k t}, Q_{i k t}, I_{i t}, S_{i k t}, \underline{b}_{k t}, \bar{b}_{k t}, \underline{B}, \bar{B} \geqslant 0, & \forall t \in \mathcal{T}, k \in \mathcal{K}, i \in \mathcal{N}_{k t}
\end{array}
$$

\subsection{Probabilistic Constraints}

As an alternative to integrating stochastic demand information in the objective function through the ESOEDC and the ERFC components, it can be included at the constraint level in the form of probabilistic constraints. Constraints 48 and 49 impose a maximum allowable probability of stock-out and route failure, respectively. Based on policy, if these constraints are imposed, the deterministic stock-out constraints 30 and the deterministic route failure constraints given by the upper bounds in constraints 36 may be dropped.

$$
\begin{array}{ll}
\mathrm{p}_{i t}^{\mathrm{DP}} \leqslant \gamma^{\mathrm{DP}}, & \forall t \in \mathcal{T}, i \in \mathcal{P} \\
\mathrm{p}_{\mathscr{S}, k}^{\mathrm{RF}} \leqslant \gamma^{\mathrm{RF}}, & \forall k \in \mathcal{K}, \mathscr{S} \in \mathfrak{S}_{k}
\end{array}
$$




\section{Application Examples}

The framework developed and presented in Sections 3, 4 and 5 can be applied to problems from different fields of routing and logistics optimization. In the sections below, we discuss in more detail a vehicle routing problem, a health care inventory routing problem, a waste collection inventory routing problem, a maritime inventory routing problem, and a facility maintenance problem.

\subsection{Vehicle Routing Problem}

In a VRP setting, the presence of stochastic demands may lead to route failures but stock-outs do not apply. To adapt the framework, we define a planning horizon $\mathcal{T}=\{0,1,2\}$, s.t. $\mathrm{H}_{k 0}=\mathrm{H}_{k 2}=0, \forall k \in \mathcal{K}$, i.e. the planning horizon consists of three periods and no vehicle is available in periods $t=0$ and $t=2$. Moreover, $I_{i 0}=\omega_{i}$ and $\mathcal{L}_{i}=\left\{\omega_{i}\right\}, \forall i \in \mathcal{P}$, i.e. the initial inventory of all demand points is equal to capacity and we apply an OU inventory policy. Given the action sequence in Definition 3 , the visits to the demand points in period $t=1$ deliver the demands $\rho_{i 0}$ of period 0 . The VRP is a single-period problem and the fact that it is effectively solved for period $t=1$ is of no consequence. In model (VRP), the objective (50) consists of the $\mathrm{RC}$ and the ERFC components. Given constraints (37) and (38), constraints (51) force a visit to a supply point immediately after the origin depot. Constraints (24) are replaced by constraints (52) below to enforce a delivery to each demand point in period $t=1$, a necessary condition for a feasible VRP solution. The periodicity related constraints 27 and 28 are dropped as they become irrelevant for a single period.

$$
\begin{array}{ll}
(\mathrm{VRP}) \min & z=\mathrm{RC}+\mathrm{ERFC} \\
\text { s.t. } \quad \text { Constraints }(18)-23), 25-26,29 & \\
& Q_{o^{\prime} k 1}=\Omega_{k}, \quad \forall k \in \mathcal{K}, o^{\prime} \in \mathcal{O}_{k 1}^{\prime} \\
& \sum_{k \in \mathcal{K}} y_{i k 1}=1, \quad \forall i \in \mathcal{P}
\end{array}
$$

\subsection{Health Care Inventory Routing Problem}

The health care IRP generalizes the nurse routing and scheduling problem, in which nurses visit patient homes to provide treatment. In this problem, $\mathcal{P}$ is the set of patient homes and $\mathcal{D}$ is the set of medical facilities. In addition to providing treatment, nurses deliver medications with stochastic demand. Continuity of care and workload balancing, which are two of the paramount concerns in the nurse routing problem, are supported by the framework. And so is the periodic aspect, given that medical treatments typically have to be performed with a certain frequency. Pricing can also be introduced in the setup via a negative visit cost. We keep the model (HCIRP) general, including all constraints, and with the objective function 53 including all but the EIHC component.

$$
\begin{array}{lll}
(\mathrm{HCIRP}) & \min & z=\mathrm{VC}+\mathrm{RC}+\mathrm{WB}+\mathrm{ESOEDC}+\mathrm{ERFC} \\
& \text { s.t. } & \text { Constraints } 18 \\
&
\end{array}
$$

\subsection{Waste Collection Inventory Routing Problem}

In this IRP variant, trucks collect waste from containers with stochastic demands. Here, $\mathcal{P}$ denotes the set of waste containers and $\mathcal{D}$ denotes the set of disposal facilities. We can apply the framework with minimal changes by relabeling the problem as the distribution of empty space. The objective function of model (WCIRP) includes the RC, ESOEDC and ERFC components. Given constraints (37) and (38), constraints (55) force a visit to a disposal facility immediately before the destination depot.

$$
\begin{array}{lll}
(\mathrm{WCIRP}) & \min & z=\mathrm{RC}+\mathrm{ESOEDC}+\mathrm{ERFC} \\
& \text { s.t. } & \text { Constraints } 18-47
\end{array}
$$




$$
Q_{o^{\prime \prime} k t}=0, \quad \forall t \in \mathcal{T}, k \in \mathcal{K}, o^{\prime \prime} \in \mathcal{O}_{k t}^{\prime \prime}
$$

Markov et al. (2016a) solve this problem in the context of recyclable waste collection from sensorized containers, using past container level information to predict future demands assuming a normal distribution of the error terms. They present a case study with rich IRP instances derived from real data from the canton of Geneva, Switzerland. The authors are able to achieve a significant reduction in the occurrence of overflows for the same routing cost compared to alternative deterministic policies. They also analyze the solution properties of a rolling horizon approach and derive empirical lower and upper bounds.

\subsection{Maritime Inventory Routing Problem}

In this problem, a fleet of ships transports a commodity from a set $\mathcal{D}$ of supply terminals to a set $\mathcal{P}$ of demand terminals. A particular feature of this application is that emergency deliveries may be impractical due to long shipping distances, which would make the state of stock-out at a demand terminal a final state. This can be achieved simply by setting the probabilities defined by expression (5) to one. Since emergency deliveries are not performed, the emergency delivery $\operatorname{cost} \zeta_{i}=0, \forall i \in \mathcal{P}$. Maritime routing problems are also characterized by open and multi-period tours, which may include idling. In our framework, constraints (18) allow for open tours, while multi-period tours are enabled by defining the set of depots so that $\exists o \in \mathcal{O}$ s.t. $\pi_{o i}=\pi_{i o}=0, \forall i \in \mathcal{P} \cup \mathcal{D}$ and $\mathcal{O}_{k t}^{\prime} \equiv \mathcal{O}_{k t}^{\prime \prime} \equiv \mathcal{O}, \forall t \in \mathcal{T}, k \in \mathcal{K}$, or in other words there is an origin and a destination depot at zero distance from each demand and supply terminal. A tour can thus effectively end at a demand or supply terminal in period $t$ and start from it in period $t+1$. The objective function of model (MIRP) includes all but the WB component. The VC component, in particular, may be used to capture terminal docking fees.

$$
\begin{array}{lll}
(\mathrm{MIRP}) & \min & z=\mathrm{EIHC}+\mathrm{VC}+\mathrm{RC}+\mathrm{ESOEDC}+\mathrm{ERFC} \\
& \text { s.t. } & \text { Constraints } 18-47
\end{array}
$$

\subsection{Facility Maintenance Problem}

The facility maintenance problem is a probability-based routing problem in which a set of facilities is visited by a set of technicians for inspection. In this problem, the set $\mathcal{P}$ represents the facilities, while the set $\mathcal{D}$ is irrelevant. Uncertainty with respect to breakdowns can be considered as accumulating in a fashion similar to that of inventory. Consider facility $i \in \mathcal{P}$ in period $t$. We can interpret the state $\sigma_{i t}=1$ as a breakdown, and the state $\sigma_{i t}=0$ as operational. If a facility is in a state of breakdown in period $t$, an emergency visit must be performed to repair it. The probability of breakdown $\mathrm{p}_{i t}^{\mathrm{DP}}$ of facility $i$ in period $t$ is adapted from expression (7) as a function of the most recent visit to the facility and is modeled as:

$$
\mathrm{p}_{i t}^{\mathrm{DP}}=\mathbb{P}\left(\sigma_{i t}=1 \mid g \in \mathbb{Z}: g<t: \exists k \in \mathcal{K}: y_{i k g}=1\right) .
$$

The use of the set $\mathbb{Z}$, which includes the negative integers, implies that the most recent visit may be before the start of the planning horizon $\mathcal{T}$. The states $\sigma_{i 0}, \forall i \in \mathcal{P}$ are known with certainty. The objective function (58) in model (FMP1) is the sum of routing cost and the Expected Emergency Repair Cost (EERC). All inventory related constraints $294-(35)$ and vehicle capacity related constraints $(36)-(39)$ are irrelevant and are hence dropped. The new set of constraints (59) is added to force a visit to a facility in a state of breakdown in period $t=0$.

$$
\begin{array}{lll}
\text { (FMP1) } & \min & z=\mathrm{RC}+\mathrm{EERC} \\
\text { s.t. } & \text { Constraints } 18-28,40-47 \\
& & \sum_{k \in \mathcal{K}} y_{i k 0}=1, \quad \forall i \in \mathcal{P}: \mathrm{p}_{i 0}^{\mathrm{DP}}=1
\end{array}
$$

The EERC is a reformulation of the ESOEDC from formula 150 and is expressed as:

$$
\mathrm{EERC}=\sum_{t \in \mathcal{T} \cup \mathcal{T}^{+}} \sum_{i \in \mathcal{P}} \mathrm{p}_{i t}^{\mathrm{DP}} \zeta_{i} .
$$


Since the probabilities in the facility maintenance problem are provided exogenously, as opposed to being calculated based on demand stochasticity, an alternative formulation involving the probabilistic constraints (48) is given in model (FMP2). Since the treatment of the probability of breakdown is in the constraints, the objective (61) is routing-only.

$$
\begin{aligned}
& \text { (FMP2) min } z=\mathrm{RC} \\
& \text { s.t. Constraints 18 -28, 40-47) } \\
& \text { Constraints 48) } \\
& \text { Constraints (59) }
\end{aligned}
$$

Given that the facility maintenance problem considers no demands, unlike in the case of the waste collection IRP, there is no deterministic equivalent problem that simply ignores the stochastic components. We could imagine several deterministic policies, for example periodicity-based visits enforced by constraints (27)-(28). A more flexible deterministic alternative would be visiting a facility $i \in \mathcal{P}$ at least $\nu_{i}$ times over the planning horizon. In the model (FMPD) below, this is ensured by constraints 62.

$$
\begin{array}{ll}
\text { (FMPD) } \min & z=\mathrm{RC} \\
\text { s.t. } \quad & \text { Constraints } 18,28,40-47 \\
& \text { Constraints }(59) \\
& \sum_{t \in \mathcal{T}} \sum_{k \in \mathcal{K}} y_{i k t} \geqslant \nu_{i}, \quad \forall i \in \mathcal{P}
\end{array}
$$

\section{Numerical Experiments}

In what follows, we analyze the key modeling and performance features of the unified framework with a series of numerical experiments. Section 7.1 describes the testbeds, namely a set of waste collection IRP instances and a set of facility maintenance instances. This is followed by Section 7.2 , which uses the first set to evaluate the framework's computational tractability and modeling realism. In particular, Section 7.2.1 studies the effect on the computation burden of using empirical distribution functions for calculating the route failure probabilities at runtime, while Section 7.2 .2 analyzes the objective function's overestimation of the real cost. Finally, Section 7.3 presents a case study based on the facility maintenance problem. Various solution methodologies can be applied to the framework, as long as they can handle the probability-based calculations and support its the rich routing features. We extend the ALNS developed by Markov et al. (2016a), which exhibits excellent performance on VRP and IRP benchmark instances from the literature, as well as on the waste collection IRP instances referred to above. The extension details are outlined in Appendix D. The ALNS is implemented as a single-thread application in Java and the calculator for the state probability trees in Figure 1 is scripted in R. All experiments have been performed on a $3.33 \mathrm{GHz}$ Intel Xeon X5680 server running a 64-bit Ubuntu 16.04.2. In the results presented below, each instance is solved 10 times.

\subsection{Instances}

The first set of instances is proposed by Markov et al. (2016a). These are 63 waste collection IRP instances built from data on white glass collections in the canton of Geneva, Switzerland. Figure 2, which is borrowed from Markov et al. (2016a), maps the collection points for recyclable materials extracted from the cantonal open data portal (SITG, 2017). Not all of these points are included in the case study, and further details are not disclosed for confidentiality reasons. The instances are created using the historical records of weekly visits for a sample period covering the years 2014, 2015 and 2016. The planning horizon is seven days

long, starting on Monday and finishing on Sunday. Moreover, according to constraints 30 there should be no expected overflows on the following Monday. On average, there are 41 containers per instance, with a maximum of 53, and their volumes range from 1000 to 3000 liters. Collection takes three or five minutes 
Figure 2: Geneva Service Area (Markov et al., 2016a)

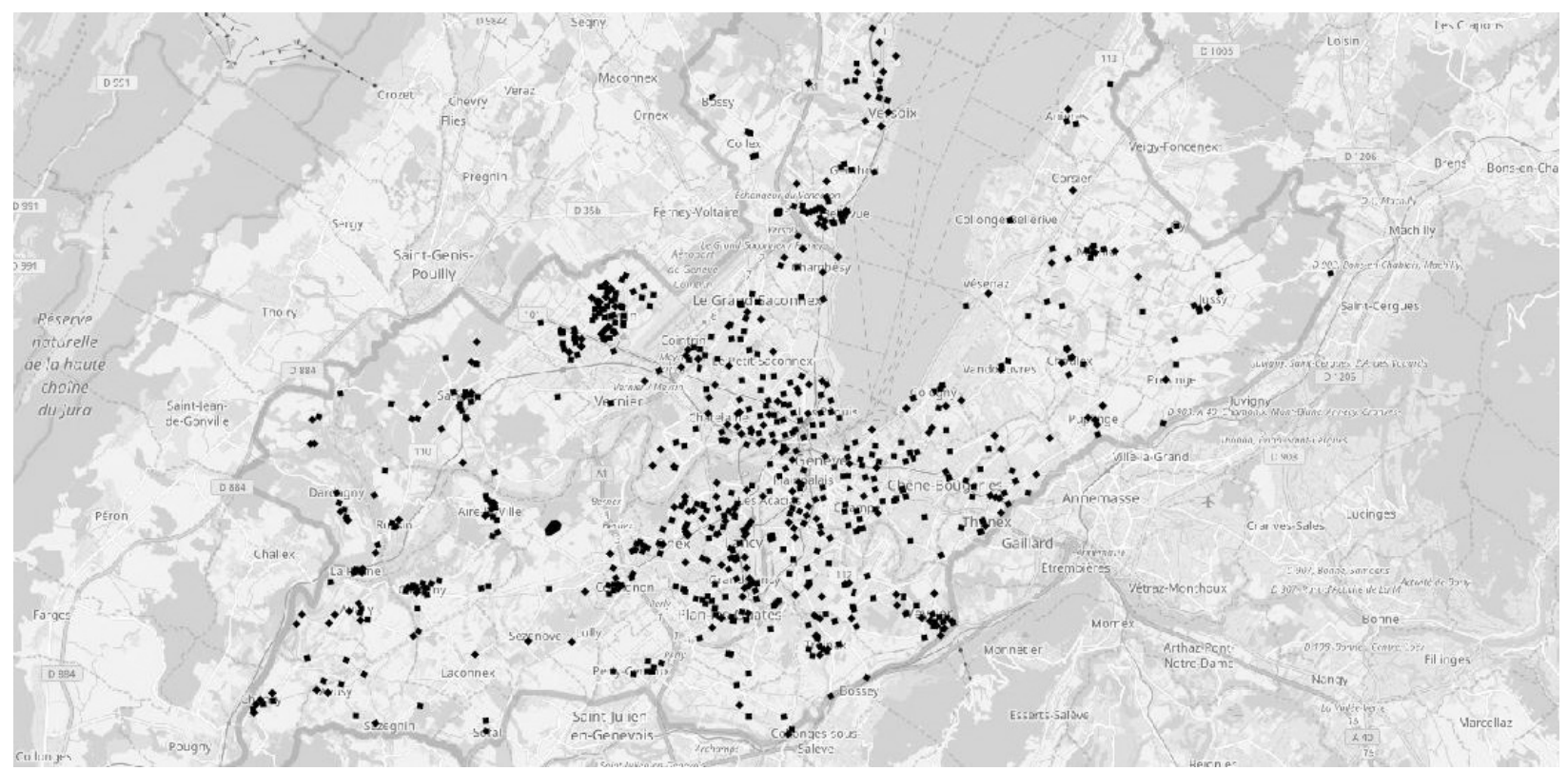

depending on container type. There are two dumps located far from each other outside the city of Geneva. The fleet consists, depending on the instance, of one or two heterogeneous vehicles with volume capacities of approximately 30,000 liters and weight capacities of 10,000 to 15,000 kg, which do not perform collections during the weekend. Demands are forecast using the count data mixture model presented in Markov et al. (2016a) using the previous 90 days of data, and assuming independent normally distributed error terms $\varepsilon_{i t}$ for all $i \in \mathcal{P}$ and $t \in \mathcal{T}$, which is supported by the data. Absence of historical container level data prevents demand forecasting for certain weeks of the sample period, for which instances are not generated. We use realistic or reasonable values for the tour duration, the time windows and the cost parameters. In particular, tours are restricted to a maximum duration of four hours each, and the time windows correspond to 8:00 a.m. until noon. For the trucks, we use a daily deployment cost of $100 \mathrm{CHF}$, a per-kilometer cost of 2.95 $\mathrm{CHF}$ and a per-hour cost of $40 \mathrm{CHF}$. We assume that the municipality charges the collector $100 \mathrm{CHF}$ for a container overflow.

The second set consists of 94 instances of the facility maintenance problem with an average of 42 facilities and a maximum of 62 . These instances are built from the same data used for building the waste collection IRP instances. However, since the facility maintenance problem described in Section 6.5 does not consider demands, we are not limited by the absence of historical container level data. Hence, the 94 instances of the facility maintenance problems vs. the 63 instances of the waste collection IRP. For each facility $i \in \mathcal{P}$, we set a service duration of 30 minutes, and tours are now constrained to a maximum duration of eight hours, instead of four. The probability of breakdown is modeled using the cumulative distribution function of the $\log$-logistic distribution. That is, the probability $\mathrm{p}_{i t}^{\mathrm{DP}}$ of breakdown of facility $i$ in period $t$ defined in formula (57) is given by:

$$
\mathrm{p}_{i t}^{\mathrm{DP}}=\frac{1}{1+\left(\frac{t-g}{\alpha}\right)^{-\beta}},
$$

where $g$ is the period of the most recent visit. We set the value of $\beta$ to 5 , while $\alpha$ is randomly chosen for each facility as an integer between 10 and 15, inclusive. Figure 3 plots the breakdown probability for the different values of the $\alpha$ parameter. In addition, for each facility in period 0 , we draw a random integer between 1 and 3, inclusive, for the number of days since the most recent visit. In effect, the probability accumulates in a way similar to how inventory builds up in the IRP. 
Figure 3: Breakdown Probabilities for Different Values of $\alpha$

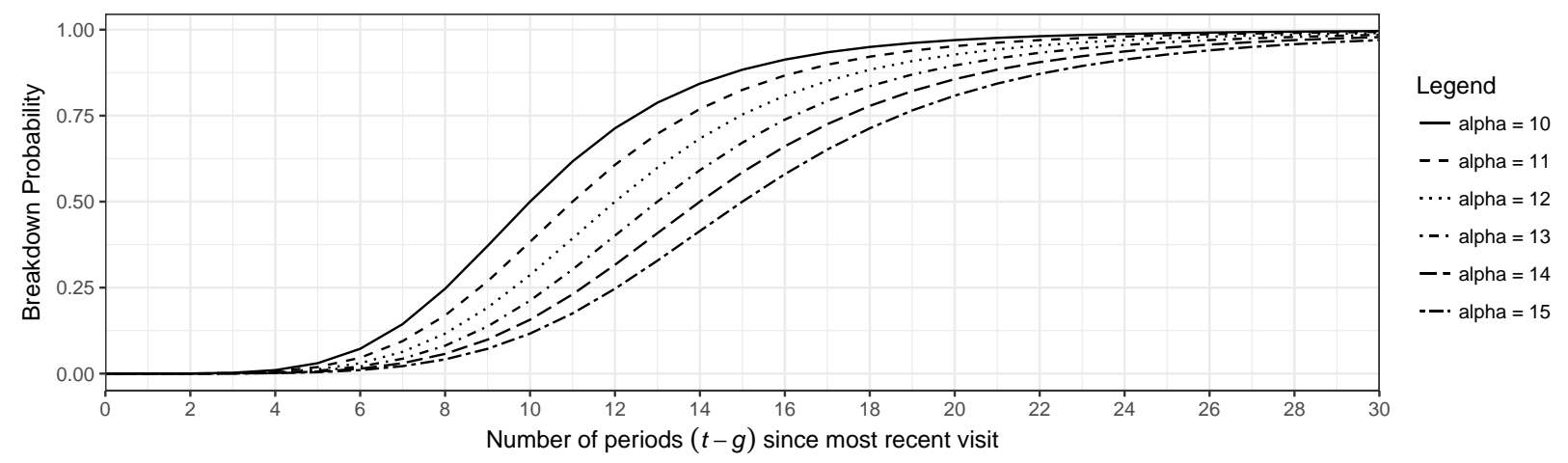

\subsection{Waste Collection Case Study}

Markov et al. (2016a) solve the waste collection IRP represented by model (WCIRP) of Section 6.3. They show that the stochastic approach significantly outperforms alternative deterministic policies often employed in practice, such as buffer capacities, in its ability to reduce the occurrence of container overflows for the same routing cost. The authors also confirm the benefits of a rolling horizon approach. Dynamically including new information at each rollover results in solutions which are on average $11 \%$ cheaper compared to those of a static problem solved for the same planning horizon. Here, we conduct further experiments on these instances. In particular, Section 7.2.1 assesses the effect on the computational burden of using empirical distribution functions at runtime for calculating the route failure probabilities. Section 7.2 .2 analyzes the objective function's overestimation of the real cost previously discussed in Section 5.1.1.

\subsubsection{Use of Empirical Distribution Functions.}

As described in Section 4.3, assuming iid error terms drawn from any distribution $\Phi$ allows the partial preprocessing of the route failure probabilities through the derivation of empirical distribution functions to be used at runtime. Clearly, the main risk of using such functions at runtime is their effect on the computation time, and hence tractability, and the precision of the resulting probability. To investigate this, we use the simulation methodology described in Appendix C to build Empirical Cumulative Distribution Functions (ECDFs) for $M=100,000$ draws. The ECDFs are constructed using the EmpiricalDistribution class of the Apache Commons Math 3.6.1 release. We test two configurations for the ECDFs, one binning the draws in 1000 bins and one binning them in 100 bins. Testing shows that the configuration with 1000 bins exhibits a squared error with respect to the normal distribution in the order of $10^{-7}$, while for the configuration with 100 bins, it is in the order of $10^{-6}$.

The normality of the error terms of the waste collection IRP instances allows us to make a meaningful comparison between analytical approximation and the use of ECDFs for the route failure probabilities. In particular, the distribution of $\Gamma_{\mathscr{S}}$ in formula (8) remains normal and can thus be analytically approximated at runtime. In parallel, the set of relevant ECDFs discussed in Section 4.3 and Appendix C can be preprocessed for the comparison. Table 2 reports the results of the experiments, which are performed for an Emergency Collection Cost (ECC) $\zeta_{i}=100$ CHF for all containers $i \in \mathcal{P}$ and a Route Failure Cost Multiplier (RFCM) $\psi=1$. In the table, each row presents averaged values over the 63 instances. The first column identifies the version of the ALNS used, i.e. the original one of Markov et al. (2016a), which uses analytical approximation, vs. the one using ECDFs. The analytical approximation is based on the following approximation of the error function:

$$
\operatorname{erf}(x) \approx 1-\left(a_{1} t+a_{2} t^{2}+\cdots+a_{5} t^{5}\right) e^{-x^{2}}, t=\frac{1}{1+p x},
$$


Table 2: Impact of Empirical Distribution Functions on Tractability

\begin{tabular}{|c|c|c|c|c|c|c|c|c|c|c|c|c|}
\hline \multirow[b]{2}{*}{ ALNS version } & \multirow[b]{2}{*}{ Bins } & \multirow[b]{2}{*}{$\mathrm{ECC}$} & \multirow[b]{2}{*}{$\mathrm{RFCM}$} & \multicolumn{3}{|c|}{ Cost (CHF) } & \multicolumn{3}{|c|}{ Runtime (s.) } & \multicolumn{3}{|c|}{ ECDF calls (millions) } \\
\hline & & & & Best & Avg & Worst & Best & Avg & Worst & Best & Avg & Worst \\
\hline Original & - & 100.00 & 1.00 & 662.65 & 666.64 & 672.87 & 870.65 & 906.84 & 936.40 & - & - & - \\
\hline ECDFs & 1000 & 100.00 & 1.00 & 662.63 & 666.74 & 673.35 & 909.06 & 948.77 & 982.68 & 52.95 & 58.90 & 65.00 \\
\hline ECDFs & 100 & 100.00 & 1.00 & 662.49 & 666.46 & 672.73 & 869.52 & 903.81 & 932.79 & 52.94 & 58.44 & 63.90 \\
\hline
\end{tabular}

where $p=0.3275911, a_{1}=0.254829592, a_{2}=-0.284496736, a_{3}=1.421413741, a_{4}=-1.453152027, a_{5}=$ 1.061405429, and whose maximum approximation error is $1.5 \times 10^{-7}$ (Abramowitz and Stegun, 1972).

The second column in Table 2 identifies the binning configuration, while the third and fourth columns show the ECC and the RFCM, which are the same for all instances. The fifth, sixth and seventh columns present the best, average and worst cost over 10 runs. In a similar fashion, the eighth, ninth and tenth columns report the best, average and worst computation time, and the eleventh, twelfth and thirteenth columns report the best, average and worst number of calls to the ECDFs over 10 runs. Expectedly, Table 2 shows that the different implementations have no impact on the solution cost. We also observe that the implementation with 100 bins has a computation time that is virtually the same as that of the original implementation. However, as mentioned before, the binning configuration with 1000 bins has a squared error which is one degree of magnitude lower, while its computation time is only about $5 \%$ higher. Therefore, this configuration may be preferable. Analytical approximation can only be used for a limited set of distributions, including the normal, for which the distribution of sums of random variables is defined and easily approximated. For all other cases, pre-processed ECDFs can be used and Table 2 provides encouraging results indicating that they preserve tractability and have a negligible impact on computation time.

\subsubsection{Overestimation of the Real Cost.}

As discussed in Section 5.1.1 the objective function overestimates the real cost because all terms, except the ESOEDC, ignore the cost effect of demand points stocking out earlier than expected. In addition, the overestimation depends on the type of reaction policy, which Definition 4 describes as the response to the recourse action by changes later in the planning horizon. To study the magnitude of this effect, we perform a simulation experiment counting the number of realized overflows. Given the final solution of each instance, we simulate 10,000 scenarios, sampling the independent normally distributed error terms $\varepsilon_{i t}$ for each container $i \in \mathcal{P}$ and each day $t \in \mathcal{T}$, and applying them to the expected demands $\mathbb{E}\left(\rho_{i t}\right)$. Then, for each scenario we analyze the effect of the realized overflows on the overestimation of the real cost.

Computing the overestimation for a do-nothing reaction policy is trivial. In the absence of inventory holding costs, the effect may only appear in the ERFC component as detailed in the proof to Proposition 2. Clearly, the overestimation will be higher for an optimal reaction policy which, in the occurrence of overflows, reoptimizes all subsequent decisions. However, computing the overestimation for an optimal reaction policy has a significant computational burden, as it requires that re-optimization be done after each overflow for the 10,000 simulated scenarios. Therefore, we consider the following intuitive upper bound on the overestimation for an optimal reaction policy. Consider a container $i$ that overflows on day $g$ and is visited for a regular collection on days $t>g$. Now, take the minimum day $h=\min t>g$ on which container $i$ is visited for a regular collection and posit an optimal reaction policy so good that it removes the cost effect of container $i$ from all days $t \geqslant h$. First, we remove the container from all tours performed on days $t \geqslant h$, thus considering the highest possible overestimation of the RC and ERFC components. Secondly, we disregard its probability of overflow on days $t \geqslant h$, thus considering the highest possible overestimation of the ESOEDC component.

Figure 4 plots the overestimation for a do-nothing reaction policy as well as the discussed upper bound on the overestimation for an optimal reaction policy of objective (54) of the waste collection IRP at the 75th, 90th, 95th and 99th percentile of the 10,000 scenarios. We present the results for an Emergency Collection Cost (ECC) $\zeta_{i}=25,50$ and $100 \mathrm{CHF}$, identical for all containers, and a Route Failure Cost Multiplier (RFCM) 
Figure 4: Objective Function's Overestimation of the Real Cost

(a) Objective Function Overestimation for ECC $=100 \mathrm{CHF}, \mathrm{RFCM}=1$
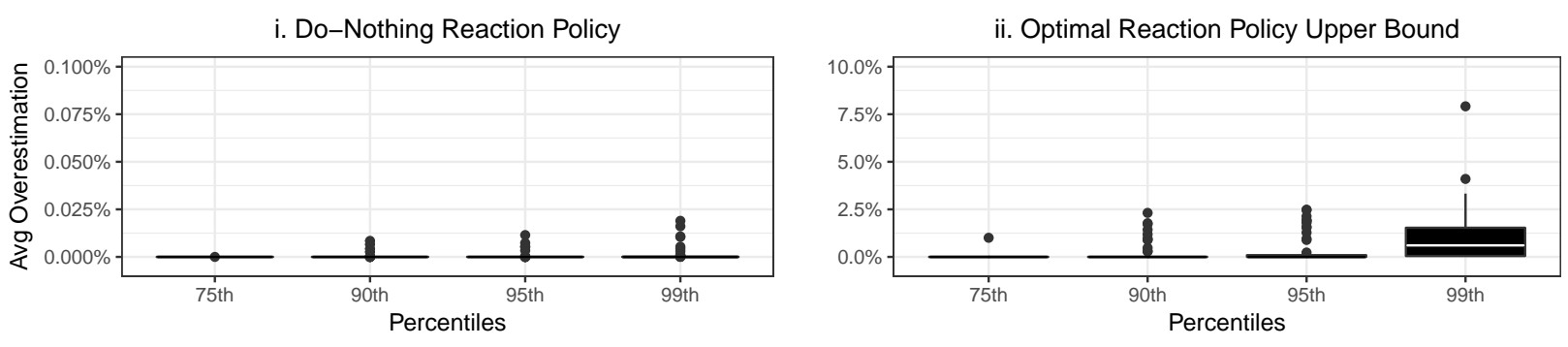

(b) Objective Function Overestimation for ECC $=50 \mathrm{CHF}, \mathrm{RFCM}=1$
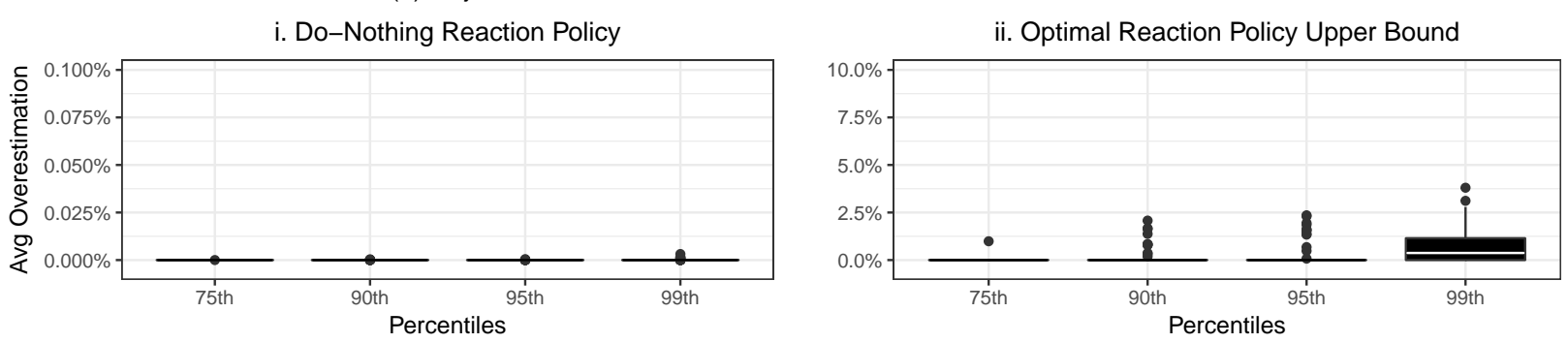

(c) Objective Function Overestimation for ECC $=25 \mathrm{CHF}, \mathrm{RFCM}=1$
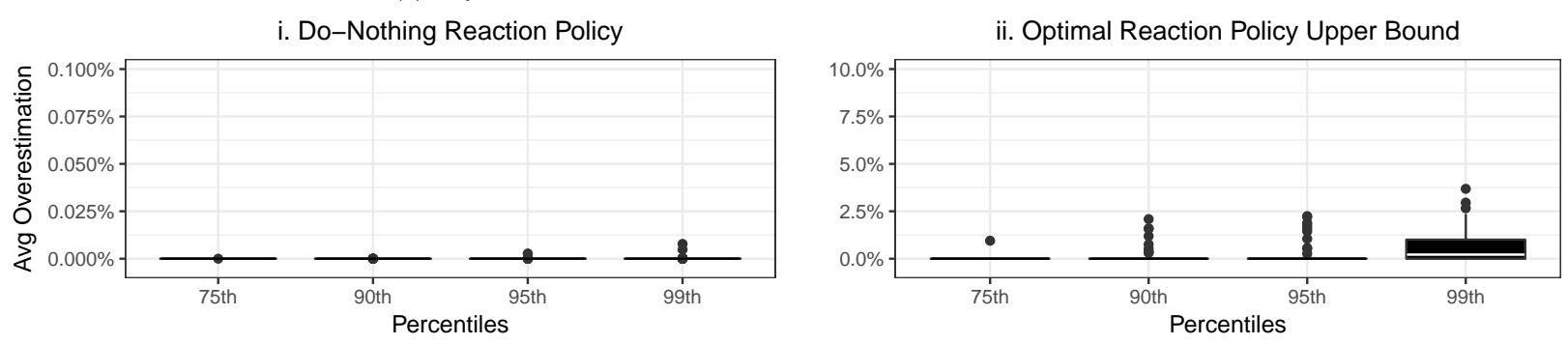

$\psi=1$. Each box-plot is constructed using the average values over 10 runs for each of the 63 instances. The overestimation for the do-nothing reaction policy is marginal, which is due to the low probability of route failure observed in general for the waste collection IRP instances. Unsurprisingly, the upper bound on the overestimation for the optimal reaction policy appears to be linked to the level of the ECC. The median upper bound is approximately zero for the 75th, 90th and 95th percentile, with the maximum values reaching $2.5 \%$. It becomes more pronounced at the 99 th percentile, where the median values are $0.61 \%, 0.37 \%$ and $0.22 \%$ for an ECC of $100 \mathrm{CHF}, 50 \mathrm{CHF}$ and $25 \mathrm{CHF}$, respectively, which indicates the generally very low level of overestimation of the real cost. The maximum values do not exceed $8 \%$ for an ECC of 100 CHF, and $4 \%$ for an ECC of $50 \mathrm{CHF}$ and $25 \mathrm{CHF}$. There is a strong correlation in the order of $70 \%$ between the number of realized overflows and the upper bound across the 63 instances. In Section 4 , we argued the importance of tractability in terms of the probability calculations that enter the objective function. Using simplification techniques in the probability expressions ignores some of the uncertainty propagation which, as proved in Proposition 2, leads to an overestimation of the real cost. Nevertheless, the results for the waste collection IRP instances indicate that this overestimation is marginal, and even a trivial upper bound on the optimal reaction policy implies a median overestimation of the real cost of less than $1 \%$. 


\subsection{Facility Maintenance Case Study}

The facility maintenance problem defined in Section 6.5 considers a set of facilities that have to be periodically inspected in order to limit the occurrence of breakdowns. Unlike the waste collection IRP, this problem does not consider demands. Thus, there is no deterministic equivalent to the stochastic problem. We start by comparing the two stochastic models proposed in Section 6.5. The models (FMP1) and (FMP2) treat uncertainty using a probabilistic objective function and probabilistic constraints, respectively. While both approaches use the same probability information, they do not use it in the same way. Specifically, the probabilistic objective approach calculates the probability of incurring the emergency repair cost and lets the model determine the best balance between the routing and the expected emergency repair cost. The breakdown probabilities in the final solution thus depend on the value of the emergency repair cost itself. The probabilistic constraints approach controls the probability of breakdown in a rather artificial way. One usually knows what it costs to perform an emergency repair, while it is unclear what a reasonable value of the maximum allowable probability of breakdown $\gamma^{\mathrm{DP}}$ should be. At any rate, while these two approaches are different modeling-wise and from a conceptual stance, they are expected to be able to produce the same range of results. To verify this, we solve the model (FMP1) for a set of Emergency Repair Cost (ERC) $\zeta_{i}$ values, where $\zeta_{i}$ is identical for each facility $i \in \mathcal{P}$, and the model (FMP2) for a set of values for $\gamma^{\mathrm{DP}}$.

The results are summarized in Tables 3 and 4 , where each line is an averaged result over the 94 instances. In both tables, the first column identifies the modeling approach, the second one reports the value of the ERC and the third one the value of the maximum allowable breakdown probability $\gamma^{\text {DP }}$. In Table 3 , the fourth column presents the average runtime in seconds, while the fifth and sixth columns report the average number of tours and facility visits, respectively. The rest of the columns report the best, average and worst results over 10 runs, and the percent gap between the average and best, and the worst and best results. Computation times are reasonable and, as expected, strongly correlated to the number of facility visits, and as a result to the cost. Not surprisingly, higher numbers of facility visits also correspond to higher numbers of tours. The gap between the average and the best solutions is in the order of 1-2\%, and the gap between the worst and the best solutions is in the order of 2-3\%, evidence of the stability of the ALNS.

Table 4 decomposes the solution cost into Routing Cost (RC) and Expected Emergency Repair Cost (EERC), whose averages are provided in the fourth and fifth columns, respectively. The last four columns are the result of a simulation experiment with 10,000 scenarios as the one described in Section 7.2.2, and report the average number of breakdowns over the 94 instances at the 75th, 90th, 95th and 99th percentile of the 10,000 scenarios. There appears to be, as expected, a clear negative correlation between the routing cost and the number of breakdowns at any percentile. This happens because higher routing costs are associated with more frequent facility visits and, as per formula (63), with lower breakdown probabilities. Moreover, we notice that the routing cost and the number of breakdowns for models (FMP1) and (FMP2) vary within

Table 3: Basic Results for Model (FMP1) vs. Model (FMP2)

\begin{tabular}{|c|c|c|c|c|c|c|c|c|c|c|}
\hline Model & ERC & $\gamma^{\mathrm{DP}}$ & Runtime (s.) & $\begin{array}{r}\text { Avg Num } \\
\text { Tours } \\
\end{array}$ & $\begin{array}{r}\text { Avg Num } \\
\text { Visits }\end{array}$ & $\begin{array}{r}\text { Best Cost } \\
(\mathrm{CHF}) \\
\end{array}$ & $\begin{array}{r}\text { Avg Cost } \\
(\mathrm{CHF}) \\
\end{array}$ & $\begin{array}{r}\text { Worst Cost } \\
(\mathrm{CHF}) \\
\end{array}$ & $\begin{array}{r}\text { Gap Avg- } \\
\text { Best (\%) }\end{array}$ & $\begin{array}{r}\text { Gap Worst- } \\
\text { Best (\%) }\end{array}$ \\
\hline (FMP1) & 1000.00 & - & 585.81 & 3.18 & 51.90 & 1810.57 & 1831.88 & 1857.70 & 1.18 & 2.60 \\
\hline (FMP1) & 500.00 & - & 558.97 & 2.98 & 45.88 & 1594.88 & 1618.29 & 1641.24 & 1.47 & 2.91 \\
\hline (FMP1) & 250.00 & - & 508.93 & 2.51 & 39.35 & 1404.90 & 1421.62 & 1443.89 & 1.19 & 2.78 \\
\hline (FMP1) & 100.00 & - & 419.05 & 1.81 & 27.19 & 1125.71 & 1139.90 & 1158.42 & 1.26 & 2.91 \\
\hline (FMP1) & 50.00 & - & 484.82 & 0.87 & 12.70 & 852.41 & 853.69 & 855.13 & 0.15 & 0.32 \\
\hline (FMP1) & 25.00 & - & 478.33 & 0.84 & 2.75 & 556.32 & 556.32 & 556.32 & 0.00 & 0.00 \\
\hline (FMP2) & - & 0.25 & 248.72 & 0.84 & 2.31 & 195.73 & 195.73 & 195.73 & 0.00 & 0.00 \\
\hline (FMP2) & - & 0.20 & 319.59 & 0.99 & 6.81 & 304.19 & 304.27 & 304.52 & 0.03 & 0.11 \\
\hline (FMP2) & - & 0.15 & 410.53 & 1.37 & 19.91 & 575.17 & 576.80 & 579.06 & 0.28 & 0.68 \\
\hline (FMP2) & - & 0.10 & 500.31 & 1.99 & 29.02 & 836.40 & 841.00 & 845.57 & 0.55 & 1.10 \\
\hline (FMP2) & - & 0.08 & 550.26 & 2.27 & 36.31 & 1003.84 & 1010.44 & 1016.76 & 0.66 & 1.29 \\
\hline (FMP2) & - & 0.05 & 584.48 & 2.62 & 41.07 & 1144.97 & 1154.82 & 1166.69 & 0.86 & 1.90 \\
\hline (FMP2) & - & 0.04 & 584.95 & 2.86 & 41.63 & 1201.01 & 1212.19 & 1226.82 & 0.93 & 2.15 \\
\hline (FMP2) & - & 0.03 & 627.74 & 2.88 & 43.83 & 1237.16 & 1249.44 & 1264.50 & 0.99 & 2.21 \\
\hline (FMP2) & - & 0.02 & 667.10 & 3.49 & 49.01 & 1438.88 & 1453.06 & 1463.91 & 0.99 & 1.74 \\
\hline
\end{tabular}


Table 4: Performance Indicators for Model (FMP1) vs. Model (FMP2)

\begin{tabular}{|c|c|c|c|c|c|c|c|c|}
\hline \multirow[b]{2}{*}{ Model } & \multirow[b]{2}{*}{ ERC } & \multirow[b]{2}{*}{$\gamma^{\mathrm{DP}}$} & \multirow[b]{2}{*}{ Avg RC (CHF) } & \multirow[b]{2}{*}{ Avg EERC (CHF) } & \multicolumn{4}{|c|}{ Avg Num Breakdowns } \\
\hline & & & & & 75th Perc. & 90th Perc. & 95th Perc. & 99th Perc. \\
\hline (FMP1) & 1000.00 & - & 1444.59 & 387.29 & 1.21 & 1.98 & 2.47 & 3.44 \\
\hline (FMP1) & 500.00 & - & 1304.26 & 314.03 & 1.76 & 2.53 & 3.13 & 4.20 \\
\hline (FMP1) & 250.00 & - & 1108.69 & 312.94 & 2.59 & 3.49 & 4.13 & 5.34 \\
\hline (FMP1) & 100.00 & - & 780.78 & 359.12 & 5.20 & 6.55 & 7.41 & 9.07 \\
\hline (FMP1) & 50.00 & - & 369.76 & 483.93 & 11.55 & 13.54 & 14.74 & 17.06 \\
\hline (FMP1) & 25.00 & - & 201.93 & 354.39 & 16.46 & 18.76 & 20.18 & 22.84 \\
\hline (FMP2) & - & 0.25 & 195.73 & 0.00 & 16.75 & 19.02 & 20.48 & 23.17 \\
\hline (FMP2) & - & 0.20 & 304.27 & 0.00 & 14.35 & 16.50 & 17.82 & 20.36 \\
\hline (FMP2) & - & 0.15 & 576.80 & 0.00 & 9.19 & 10.97 & 12.07 & 14.18 \\
\hline (FMP2) & - & 0.10 & 841.00 & 0.00 & 5.62 & 6.98 & 7.85 & 9.58 \\
\hline (FMP2) & - & 0.08 & 1010.44 & 0.00 & 3.91 & 5.06 & 5.84 & 7.29 \\
\hline (FMP2) & - & 0.05 & 1154.82 & 0.00 & 2.53 & 3.48 & 4.11 & 5.31 \\
\hline (FMP2) & - & 0.04 & 1212.19 & 0.00 & 2.17 & 3.06 & 3.58 & 4.75 \\
\hline (FMP2) & - & 0.03 & 1249.44 & 0.00 & 2.01 & 2.82 & 3.41 & 4.52 \\
\hline (FMP2) & - & 0.02 & 1453.06 & 0.00 & 1.22 & 2.02 & 2.47 & 3.43 \\
\hline
\end{tabular}

similar ranges. This is confirmed by Figure 5 , which is a visual representation of the above results. It demonstrates that the two approaches are logically equivalent, with similar routing costs corresponding to similar levels of occurrence of breakdowns. We stress again that both approaches are probabilistic, using the same uncertainty information in different ways.

To complete the picture, we compare the two probabilistic models to model (FMPD) of Section 6.5. which is a flexible deterministic approach oblivious to any uncertainty information. It considers a routing-only objective function and stipulates that each facility $i \in \mathcal{P}$ must be visited at least $\nu_{i}$ times during the

Figure 5: Comparison of Routing Cost and Breakdowns for Model (FMP1) vs. Model (FMP2)

(a) Routing Cost

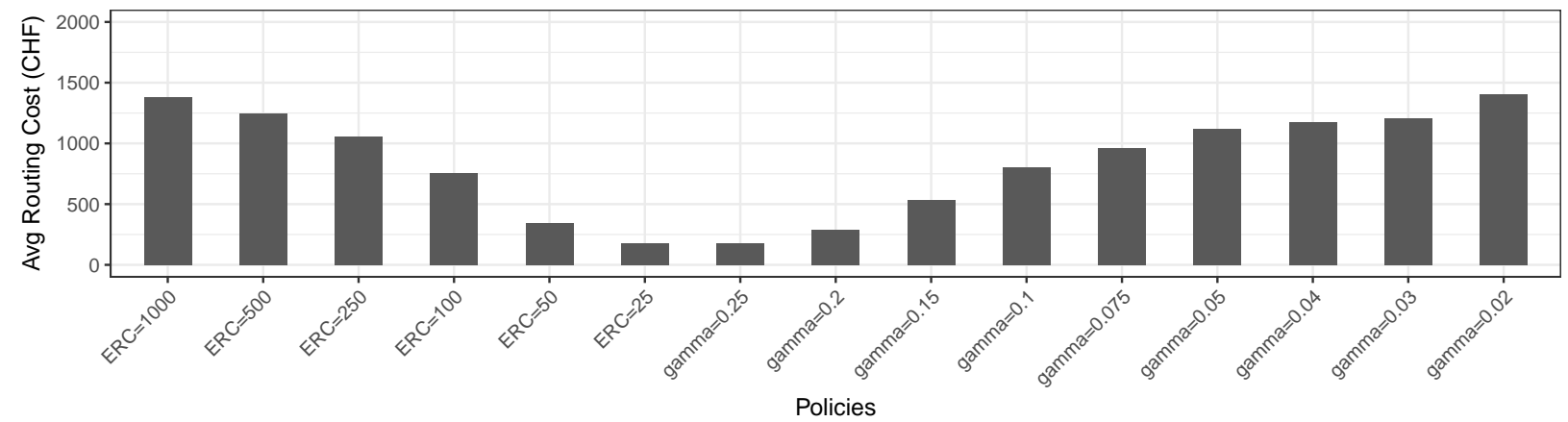

(b) Breakdowns

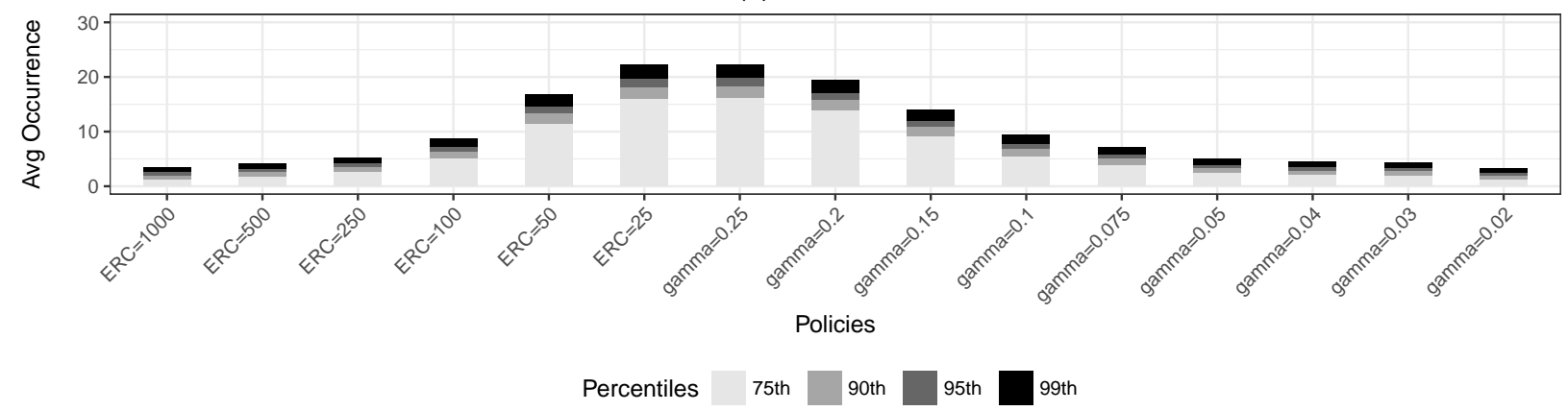


Table 5: Performance Indicators for Model (FMPD)

\begin{tabular}{lrrrrrrrr}
\hline & & & & \multicolumn{4}{c}{ Avg Num Breakdowns } \\
\cline { 5 - 8 } Model & ERC & $\nu_{i}$ & Avg RC (CHF) & Avg EERC (CHF) & 75th Perc. & 90th Perc. & 95th Perc. & 99th Perc. \\
\hline (FMPD) & - & 2 & 1945.96 & 0.00 & 3.16 & 4.10 & 4.56 & 5.71 \\
(FMPD) & - & 1 & 1140.10 & 0.00 & 4.28 & 5.47 & 6.26 & 7.77 \\
\hline
\end{tabular}

planning horizon. Table 5, which is structured in the same way as Table 4 summarizes the results for $\nu_{i}$ $=1$ and 2, with $\nu_{i}$ identical for all $i \in \mathcal{P}$. Some of the instances become infeasible for higher values of $\nu_{i}$. Based on the above information, Figure 6 presents a side-by-side comparison of the studied probabilistic and deterministic policies. Part (a) of the figure plots the number of breakdowns at the 99th percentile of each deterministic policy from Table 5 and the closest outperforming probabilistic policy from Table 4 Part (b), in turn, compares the corresponding routing costs. Overall, the comparison reveals that given a deterministic policy, we can find a probabilistic one that leads to fewer breakdowns and is at the same time cheaper, thus highlighting the clear superiority of the stochastic modeling approaches. In the analysis above, we disregard the EERC component from the comparison, as it merely provides a cost dimension to the number of expected breakdowns. In effect, we compare the two policy types in terms of the number of realized breakdowns after subjecting them to the same simulation experiment.

Figure 6: Comparison of probabilistic and deterministic policies

(a) Breakdowns at 99th percentile

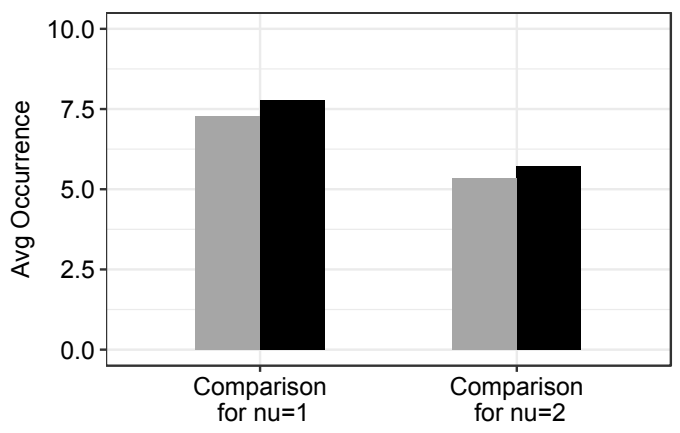

(b) Routing Cost

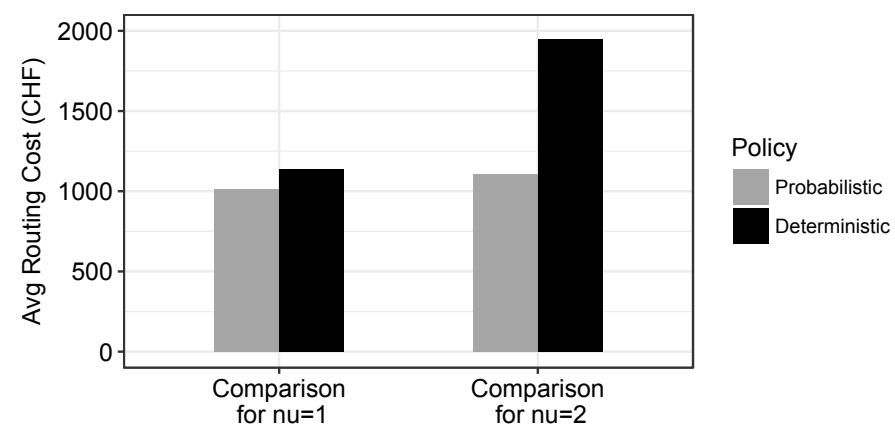

\section{Conclusion}

This work introduces, formulates and analyzes a unified framework for various classes of rich routing problems, including among others the VRP and the IRP. Demand is stochastic, can be non-stationary, and is forecast with any model that provides the expected demands over the planning horizon and the error term distribution, where the latter can be any theoretical or empirical distribution that can be simulated. We explicitly model undesirable events and include a variety of features relevant to real-world routing problems, such as multiple depots, open and multi-period tours, intermediate facilities, time windows, accessibility restrictions, visit periodicities and service choice, etc. The practical applicability of the approach is reinforced by the fact that the probability information related to demand stochasticity can be pre-computed or at least partially pre-processed. Thus, we can preserve tractability, which is critical for operational problems such as waste collection, facility maintenance, and other discussed in the text. Finally, we show that certain problems where the inventory component is not present, such as facility maintenance, can still be viewed through the prism of inventory routing, with event probabilities at the demand points, or breakdown probabilities in this specific example, accumulating as would inventory. The case study is based on realistic instances of the waste collection IRP and the facility maintenance problem. We demonstrate the tractable calculation of the route failure probabilities, which is possible for iid error terms from any distribution using pre-processed ECDFs. Using a simulation approach, we also verify that the effect of our assumptions and 
modeling simplifications in terms of overestimation of the real cost is marginal. Finally, we observe that our framework significantly outperforms alternative deterministic policies in its ability to limit the number of undesirable events for the same routing cost.

The future research directions can be classified into those of mainly practical interest and those of mainly theoretical interest. Starting from the first group, in our view the most important task is the development of additional benchmark instances, which will allow us to test the framework's full capabilities on different problem types. While there exist benchmark instances for many of the problems modeled in Section 6, they are largely deterministic or involve simple routing structures. Thus, it is crucial that the instances be based on real data. We are interested in evaluating how the framework performs on concrete problems faced by real actors in the transportation domain. Another practically relevant research idea concerns information availability. In our framework, demand is modeled at discrete time periods and inventory is updated at the start of each period. Yet, visiting a customer at different times during the same period would probably imply different delivery quantities. Real-time, or online, optimization can be used to deal with continuous time demands. In this setup, the system is updated after each customer visit and the subsequent decisions are fully or partially re-optimized based on the latest available information.

This leads us to the second group of future work directions - those of primarily theoretical interest. From a general standpoint, our framework is the first step in designing even more comprehensive objective functions that account for more complex uncertainty propagations. This should make it possible to relax some of the assumptions and allow for increasingly complex routing structures, for example including multiple commodities and compartmentalization (Mendoza et al., 2011). The integration of other stochastic elements, in particular travel and service times, is also extremely relevant, especially in application areas such as maritime routing. Finding the balance between modeling realism and the preservation of tractability is one of the main challenges in this direction. In terms of solution methodology, an important area of future research is the development of theoretical lower bounds. Our optimization models rely on arc-based formulations which are known to provide weak lower bounds (Semet et al. 2014, Poggi and Uchoa, 2014). Such models quickly become intractable even for moderate instance sizes. A promising direction is the development of a path-based formulation and a state-of-the-art column generation procedure. While this is rather straightforward for the linear and deterministic case where the pricing problem is an Elementary Shortest Path Problem with Resource Constraints (ESPPRC), the non-linear nature of our objective function will certainly pose challenges in this regard. Certain simplifications in the routing structure and approximations like demand discretization may be sufficient to linearize the objective function and cast the pricing problem as an ESPPRC. Alternatively, a more complicated pricing problem may need to be modeled and solved. Either way, the ultimate goal is the evaluation of the quality of the ALNS or any other meta-heuristic methodology applied to the framework.

\section{A Equivalence of Stock-out and Overflow Probabilities}

At the demand point level, the undesirable event for a distribution problem is a stock-out while for a collection problem it is an overflow. Given that collection can be seen as the distribution of empty space, these two events are modeled equivalently.

Proposition 3. The calculation of the probability of overflow for a collection problem is identical to the calculation of the probability of stock-out for a distribution problem.

Proof. Let $\Lambda_{i g}^{\prime}$ denote the inventory after a regular collection of demand point $i$ in period $g$. This collection is accompanied by a corresponding delivery of empty space. Thus, the empty space inventory after a regular delivery is $\Lambda_{i g}=\left(\omega_{i}-\Lambda_{i g}^{\prime}\right)$, where $\omega_{i}$ is the capacity of demand point $i$. Given a regular collection in period $g$, the unconditional probability of overflow of demand point $i$ in period $g+1$ is expressed as:

$$
\mathbb{P}\left(\Lambda_{i g}^{\prime}+\rho_{i g} \geqslant \omega_{i}\right)=\mathbb{P}\left(\left(\omega_{i}-\Lambda_{i g}^{\prime}\right)-\rho_{i g} \leqslant 0\right)=\mathbb{P}\left(\Lambda_{i g}-\rho_{i g} \leqslant 0\right),
$$

the last expression being equivalent to expression (3) for a distribution problem. Given a regular collection 
in period $g$, the conditional probability of overflow in periods later than $g+1$ is expressed as:

$$
\begin{gathered}
\mathbb{P}\left(\Lambda_{i g}^{\prime}+\sum_{t=g}^{h} \rho_{i t} \geqslant \omega_{i} \mid \Lambda_{i g}^{\prime}+\sum_{t=g}^{h-1} \rho_{i t}<\omega_{i}\right)= \\
\mathbb{P}\left(\left(\omega_{i}-\Lambda_{i g}^{\prime}\right)-\sum_{t=g}^{h} \rho_{i t} \leqslant 0 \mid\left(\omega_{i}-\Lambda_{i g}^{\prime}\right)-\sum_{t=g}^{h-1} \rho_{i t}>0\right)= \\
\mathbb{P}\left(\Lambda_{i g}-\sum_{t=g}^{h} \rho_{i t} \leqslant 0 \mid \Lambda_{i g}-\sum_{t=g}^{h-1} \rho_{i t}>0\right), \quad \forall h>g,
\end{gathered}
$$

the last expression being equivalent to expression (4) for a distribution problem. The proofs for the unconditional and conditional probabilities of overflow given an emergency collection in period $g^{\prime}>g$ follow as special cases.

\section{B Pre-computing the Stock-out Probabilities}

To pre-compute the unconditional and conditional probabilities of stock-out (3)-(6), choose a sufficiently large number $M$ and for $m \in\{1, \ldots, M\}$ simulate:

$$
\mathbf{e}^{m}=\left(e_{11}^{m}, \cdots, e_{1|\mathcal{T}|}^{m}, e_{21}^{m}, \cdots, e_{|\mathcal{P} \| \mathcal{T}|}^{m}\right)
$$

by drawing $\varepsilon$ from $\Phi$, where $\varepsilon$ is the vector of error terms defined by equation (2). Using the result of (B.1), the probability in formula (3) is pre-computed as:

$$
\mathbb{P}\left(\Lambda_{i g}-\rho_{i g} \leqslant 0\right)=\mathbb{P}\left(\varepsilon_{i g} \geqslant \Lambda_{i g}-\mathbb{E}\left(\rho_{i g}\right)\right)=\frac{\sum_{m=1}^{M} \operatorname{IF}\left(e_{i t}^{m} \geqslant \Lambda_{i g}-\mathbb{E}\left(\rho_{i g}\right), 1,0\right)}{M} .
$$

Using the same technique, the probability in formula (4) develops and pre-computes as:

$$
\begin{gathered}
\mathbb{P}\left(\Lambda_{i g}-\sum_{t=g}^{h} \rho_{i t} \leqslant 0 \mid \Lambda_{i g}-\sum_{t=g}^{h-1} \rho_{i t}>0\right)= \\
=\mathbb{P}\left(\sum_{t=g}^{h} \varepsilon_{i t} \geqslant \Lambda_{i g}-\sum_{t=g}^{h} \mathbb{E}\left(\rho_{i t}\right) \mid \sum_{t=g}^{h-1} \varepsilon_{i t}<\Lambda_{i g}-\sum_{t=g}^{h-1} \mathbb{E}\left(\rho_{i t}\right)\right)= \\
=\frac{\mathbb{P}\left(\sum_{t=g}^{h} \varepsilon_{i t} \geqslant \Lambda_{i g}-\sum_{t=g}^{h} \mathbb{E}\left(\rho_{i t}\right), \sum_{t=g}^{h-1} \varepsilon_{i t}<\Lambda_{i g}-\sum_{t=g}^{h-1} \mathbb{E}\left(\rho_{i t}\right)\right)}{\mathbb{P}\left(\sum_{t=g}^{h-1} \varepsilon_{i t}<\Lambda_{i g}-\sum_{t=g}^{h-1} \mathbb{E}\left(\rho_{i t}\right)\right)}= \\
=\frac{\sum_{m=1}^{M} \operatorname{IF}\left(\sum_{t=g}^{h} e_{i t}^{m} \geqslant \Lambda_{i g}-\sum_{t=g}^{h} \mathbb{E}\left(\rho_{i t}\right) \operatorname{AND} \sum_{t=g}^{h-1} e_{i t}^{m}<\Lambda_{i g}-\sum_{t=g}^{h-1} \mathbb{E}\left(\rho_{i t}\right), 1,0\right)}{\sum_{m=1}^{M} \operatorname{IF}\left(\sum_{t=g}^{h-1} e_{i t}^{m}<\Lambda_{i g}-\sum_{t=g}^{h-1} \mathbb{E}\left(\rho_{i t}\right), 1,0\right)} \\
\forall h>g .
\end{gathered}
$$

The function IF ([condition], 1,0) is equal to 1 if the condition is satisfied, and to 0 otherwise. The probabilities in formulas (5) and (6) are pre-computed as special cases of (B.2) and (B.3), respectively. The time complexity of calculating each probability is linear in $M$. 


\section{Partially Pre-processing the Route Failure Probabilities}

Formula (99), which defines the probability of route failure, develops as:

$$
\begin{gathered}
\mathbb{P}\left(\Gamma_{\mathscr{S}}>\Omega_{k}\right)= \\
=\mathbb{P}\left(\mathbb{E}\left(\Gamma_{\mathscr{S}}\right)+\mathcal{E}>\Omega_{k}\right)= \\
=\mathbb{P}\left(\mathcal{E}>\Omega_{k}-\mathbb{E}\left(\Gamma_{\mathscr{S}}\right)\right),
\end{gathered}
$$

where the cumulative error term $\mathcal{E}$ is derived from the definition of the delivery quantity $\Gamma_{\mathscr{S}}$ in trip $\mathscr{S}$ in formula (8) as follows:

$$
\mathcal{E}=\sum_{t \in \mathcal{T} \backslash 0} \sum_{\mathcal{S}_{t} \in \mathscr{S}} \sum_{s \in \mathcal{S}_{t}} \sum_{h=m}^{t-1} \varepsilon_{s h} .
$$

In the general case, the distribution of $\mathcal{E}$ is unknown. And while probabilities (C.1) can be approximated using the simulation techniques presented in Appendix B, the number of combinations involving different periods, demand points and discrete inventory levels is prohibitive for them to be pre-computed.

Imposing Assumption 2 allows the partial pre-processing of the route failure probabilities. The distribution of $\mathcal{E}$ now only depends on the number of iid error terms $\varepsilon_{s h}$ summed in expression (C.2), which is bounded by $N=|\mathcal{P}|(|\mathcal{T}|-1)$ as discussed in Section 4.3. Pre-processing is performed by choosing a sufficiently large number $M$ and for $m \in\{1, \ldots, M\}$ simulating:

$$
e_{g}^{m}=\sum_{t=1}^{g} \varepsilon_{i t}, \quad \forall g \in\{1, \ldots, N\}
$$

by drawing $\varepsilon_{i t}$ from the marginal distribution $\Phi^{\prime}$ for any $i \in \mathcal{P}$ (see Section 4.3). Using the result of C.3, we derive an empirical distribution function $\Phi_{g}^{\mathrm{emp}}$ of the values $\left\{e_{g}^{1}, \ldots, e_{g}^{M}\right\}, \forall g \in\{1, \ldots, N\}$. Given Assumption 2 and formulation $(\overline{C .3}), \exists g \in\{1, \ldots, N\}$ s.t. $\mathcal{E} \sim \Phi_{g}^{\mathrm{emp}}$. These empirical distribution functions are then used at runtime to calculate the probabilities in formula (C.1).

\section{ALNS Extensions}

The ALNS used for the numerical experiments in Section 7 applies the parametric configuration of Markov et al. (2016a). Section D.1 below describes the changes to the solution representation of the original implementation while Section D.2 lists three new repair operators to handle the additional routing features.

\section{D.1 Solution Representation}

With respect to the deterministic constraints, we consider all six types of feasibility violations presented in Markov et al. (2016a) plus a violation of the visit period combination constraints (28). Some of the former are redefined in view of the more general formulation and the representative distribution context. The new solution representation also considers the violations of the probabilistic constraints (48) and (49). Given a solution $s$ and the shorthand notation $(x)^{+}=\max \{0, x\}$, the feasibility violations are summarized as:

1. Vehicle capacity violation $V^{\Omega}(s)$ is redefined to capture the more general concept of trips in the framework, with trips being supply point delimited demand point sequences, possibly spanning over multiple periods. It is the sum of trip delivery quantities in excess of vehicle capacities:

$$
V^{\Omega}(s)=\sum_{k \in \mathcal{K}} \sum_{\mathscr{S} \in \mathfrak{S}_{k}}\left(\sum_{t \in \mathcal{T}} \sum_{\mathcal{S}_{t} \in \mathscr{S}} \sum_{s \in \mathcal{S}_{t}} q_{s k t}-\Omega_{k}\right)^{+} .
$$


2. Time window violation $V^{\mu}(s)$ remains unchanged. It is the sum of the upper time window bound violations of the visited points.

3. Duration violation $V^{\mathrm{H}}(s)$ is redefined to reflect the presence of multiple origin and destination depots. It is the sum of excess tour durations:

$$
V^{\mathrm{H}}(s)=\sum_{t \in \mathcal{T}} \sum_{k \in \mathcal{K}}\left(\sum_{o^{\prime \prime} \in \mathcal{O}_{k t}^{\prime \prime}} S_{o^{\prime \prime} k t}-\sum_{o^{\prime} \in \mathcal{O}_{k t}^{\prime}} S_{o^{\prime} k t}-\mathrm{H}\right)^{+} .
$$

4. Demand point violation $V^{\omega}(s)$ is redefined for a distribution context. It is the sum of negative demand point inventories over $\mathcal{T}^{+}$:

$$
V^{\omega}(s)=\sum_{t \in \mathcal{T}^{+}} \sum_{i \in \mathcal{P}}\left(-I_{i t}\right)^{+}
$$

5. Backorder limit violation $V^{0}(s)$ is also redefined for a distribution context. It is the sum of negative inventories in period $t=0$ of the demand points that are not visited in period $t=0$ :

$$
V^{0}(s)=\sum_{i \in \mathcal{P}}\left(\left(1-\sum_{k \in \mathcal{K}} y_{i k 0}\right)\left(-I_{i 0}\right)^{+}\right) .
$$

6. Accessibility violation $V^{\alpha}(s)$ remains unchanged. It is the sum of the inaccessible point visits.

7. Visit period combination violation $V^{r}(s)$ is the sum of visits performed when not required or not performed when required by the assigned visit period combinations:

$$
V^{r}(s)=\sum_{t \in \mathcal{T}} \sum_{i \in \mathcal{P}}\left|\sum_{k \in \mathcal{K}} y_{i k t}-\sum_{r \in \mathcal{C}_{i}} \alpha_{r t} c_{i r}\right| .
$$

8. Maximum stock-out probability violation $V^{\mathrm{DP}}(s)$ is the sum of stock-out probabilities in excess of the maximum allowable probability of stock-out:

$$
V^{\mathrm{DP}}(s)=\sum_{t \in \mathcal{T}} \sum_{i \in \mathcal{P}}\left(\mathrm{p}_{i t}^{\mathrm{DP}}-\gamma^{\mathrm{DP}}\right)^{+}
$$

9. Maximum route failure probability violation $V^{\mathrm{RF}}(s)$ is the sum of route failure probabilities in excess of the maximum allowable probability of route failure:

$$
V^{\mathrm{RF}}(s)=\sum_{k \in \mathcal{K}} \sum_{\mathscr{S} \in \mathfrak{S}_{k}}\left(\mathrm{p}_{\mathscr{S}, k}^{\mathrm{RF}}-\gamma^{\mathrm{RF}}\right)^{+}
$$

With the above violations, the complete solution cost during the search is represented by:

$$
\begin{gathered}
f(s)=z(s)+L^{\Omega} V^{\Omega}(s)+L^{\mu} V^{\mu}(s)+L^{\mathrm{H}} V^{\mathrm{H}}(s)+L^{\omega} V^{\omega}(s)+L^{0} V^{0}(s)+L^{\alpha} V^{\alpha}(s) \\
+L^{r} V^{r}(s)+L^{\mathrm{DP}} V^{\mathrm{DP}}(s)+L^{\mathrm{RF}} V^{\mathrm{RF}}(s),
\end{gathered}
$$

where parameters $L^{\Omega}$ through $L^{\mathrm{RF}}$ penalize each type of feasibility violation.

\section{D.2 Operators}

The following three repair operators are added to the original list of repair operators: 
1. Replace a destination depot: This operator selects a random tour and replaces its destination depot with a random destination depot $o \in \mathcal{O}_{k t}^{\prime \prime}$, where $t \in \mathcal{T}$ is the period in which the tour is performed and $k \in \mathcal{K}$ is the vehicle performing it. The algorithm then finds $\min h>t$ s.t. $\mathrm{H}_{k h}>0$, i.e. the next period $h$ for which vehicle $k$ is available, and changes the origin depot of the tour that vehicle $k$ executes in period $h$ to $o$.

2. Change visit period combination: This operator selects a random demand point $i \in \mathcal{P}$ and assigns to it a random visit period combination $r \in \mathcal{C}_{i}$.

3. Change inventory level after delivery: This operator selects a random tour executed in period $t$ and a random demand point $i$ in this tour. It then selects a random level $r \in \mathcal{L}_{i}$ s.t. $\Lambda_{i t}=r \ell_{i r t}>I_{i t}$ and assigns it to demand point $i$ in period $t$.

\section{Acknowledgment}

This research did not receive any specific grant from funding agencies in the public, commercial, or not-forprofit sectors. The authors would like to thank Matthieu de Lapparent for his advice on probability, statistics and simulation related topics and Maria Grazia Speranza for pointing out several pertinent references on the IRP.

\section{References}

Abramowitz, M. and Stegun, I. A., editors (1972). Handbook of Mathematical Functions with Formulas, Graphs, and Mathematical Tables. New York: Dover Publications.

Adelman, D. (2004). A price-directed approach to stochastic inventory/routing. Operations Research, $52(4): 499-514$.

Aghezzaf, E.-H. (2008). Robust distribution planning for supplier-managed inventory agreements when demand rates and travel times are stationary. The Journal of the Operational Research Society, 59(8):10551065.

Andersson, H., Hoff, A., Christiansen, M., Hasle, G., and Løkketangen, A. (2010). Industrial aspects and literature survey: Combined inventory management and routing. Computers $\& 5$ Operations Research, $37(9): 1515-1536$.

Archetti, C., Bertazzi, L., Paletta, G., and Speranza, M. G. (2011). Analysis of the maximum level policy in a production-distribution system. Computers $\mathscr{E}$ Operations Research, 38(12):1731-1746.

Arslan, A. N. and Papageorgiou, D. J. (2015). Bulk ship fleet renewal and deployment under uncertainty: A multi-stage stochastic programming approach. Working Paper, Department of Industrial \& Systems Engineering, University of Florida, Gainesville, FL, USA.

Bell, W. J., Dalberto, L. M., Fisher, M. L., Greenfield, A. J., Jaikumar, R., Kedia, P., Mack, R. G., and Prutzman, P. J. (1983). Improving the distribution of industrial gases with an on-line computerized routing and scheduling optimizer. Interfaces, 13(6):4-23.

Berman, O. and Larson, R. C. (2001). Deliveries in an inventory/routing problem using stochastic dynamic programming. Transportation Science, 35(2):192-213.

Bertazzi, L., Paletta, G., and Speranza, M. G. (2002). Deterministic order-up-to level policies in an inventory routing problem. Transportation Science, 36(1):119-132.

Bertsimas, D. and Sim, M. (2003). Robust discrete optimization and network flows. Mathematical Programming, 98(1-3):49-71. 
Bertsimas, D. and Sim, M. (2004). The price of robustness. Operations Research, 52(1):35-53.

Bitsch, B. (2012). Inventory routing with stochastic demand. Master's thesis, Aarhus School of Business and Social Sciences, Aarhus University, Aarhus, Denmark.

Campbell, A., Clarke, L., Kleywegt, A., and Savelsbergh, M. (1998). The inventory routing problem. In Crainic, T. G. and Laporte, G., editors, Fleet Management and Logistics, Centre for Research on Transportation, pages $95-113$. Springer US.

Cheng, L. and Duran, M. A. (2004). Logistics for world-wide crude oil transportation using discrete event simulation and optimal control. Computers \& Chemical Engineering, 28(6-7):897-911.

Coelho, L. C., Cordeau, J.-F., and Laporte, G. (2014). Thirty years of inventory routing. Transportation Science, 48(1):1-19.

Cordeau, J.-F., Gendreau, M., and Laporte, G. (1997). A tabu search heuristic for periodic and multi-depot vehicle routing problems. Networks, 30(2):105-119.

Dantzig, G. and Ramser, R. (1959). The truck dispatching problem. Management Science, 6:80-91.

Delage, E. and Iancu, D. A. (2015). Robust multistage decision making. In The Operations Research Revolution, INFORMS Tutorials in Operations Research, pages 20-46.

Dror, M. and Trudeau, P. (1986). Stochastic vehicle routing with modified savings algorithm. European Journal of Operational Research, 23(2):228-235.

Errarhout, A., Kharraja, S., and Corbier, C. (2016). Two-stage stochastic assignment problem in the home health care. IFAC-PapersOnLine, 49(12):1152-1157. 8th IFAC Conference on Manufacturing Modelling, Management and Control MIM, Troyes, France, June 28-30, 2016.

Errarhout, A., Kharraja, S., and Matta, A. (2014). The uncertainty in the home health care assignment problem. In Proceedings of the 3rd International Conference on Operations Research and Enterprise Systems, pages 453-459, Angers, France, March 6-8, 2014.

Gendreau, M., Jabali, O., and Rei, W. (2014). Chapter 8: Stochastic vehicle routing problems. In Toth, P. and Vigo, D., editors, Vehicle Routing: Problems, Methods, and Applications, Second Edition. SIAM.

Gendreau, M., Jabali, O., and Rei, W. (2016). 50th anniversary invited article-Future research directions in stochastic vehicle routing. Transportation Science, 50(4):1163-1173.

Gounaris, C. E., Wiesemann, W., and Floudas, C. A. (2013). The robust capacitated vehicle routing problem under demand uncertainty. Operations Research, 61(3):677-693.

Hemmelmayr, V., Doerner, K. F., Hartl, R. F., and Savelsbergh, M. W. (2010). Vendor managed inventory for environments with stochastic product usage. European Journal of Operational Research, 202(3):686695.

Hvattum, L. M. and Løkketangen, A. (2009). Using scenario trees and progressive hedging for stochastic inventory routing problems. Journal of Heuristics, 15(6):527-557.

Hvattum, L. M., Løkketangen, A., and Laporte, G. (2009). Scenario tree-based heuristics for stochastic inventory-routing problems. INFORMS Journal on Computing, 21(2):268-285.

Johansson, O. M. (2006). The effect of dynamic scheduling and routing in a solid waste management system. Waste Management, 26(8):875-885.

Kleywegt, A. J., Nori, V. S., and Savelsbergh, M. W. P. (2002). The stochastic inventory routing problem with direct deliveries. Transportation Science, 36(1):94-108.

Kleywegt, A. J., Nori, V. S., and Savelsbergh, M. W. P. (2004). Dynamic programming approximations for a stochastic inventory routing problem. Transportation Science, 38(1):42-70. 
Lahyani, R., Khemakhem, M., and Semet, F. (2015). Rich vehicle routing problems: From a taxonomy to a definition. European Journal of Operational Research, 241(1):1-14.

Lanzarone, E. and Matta, A. (2009). Value of perfect information in home care human resource planning with continuity of care. In Proceedings of the 35th Conference on Operational Research Applied to Health Services (ORAHS 2009), Leuven, Belgium, July 13-17, 2009.

Lanzarone, E. and Matta, A. (2012). A cost assignment policy for home care patients. Flexible Services and Manufacturing Journal, 24(4):465-495.

Lanzarone, E., Matta, A., and Sahin, E. (2012). Operations management applied to home care services: The problem of assigning human resources to patients. IEEE Transactions on Systems, Man, and Cybernetics - Part A: Systems and Humans, 42(6):1346-1363.

Louveaux, F. (1998). An introduction to stochastic transportation models. In Labbé, M., Laporte, G., Tanczos, K., and Toint, P., editors, Operations Research and Decision Aid Methodologies in Traffic and Transportation Management, pages 244-263. Springer Berlin Heidelberg.

Markov, I., Bierlaire, M., Cordeau, J.-F., Maknoon, Y., and Varone, S. (2016a). Inventory routing with non-stationary stochastic demands. Technical Report TRANSP-OR 160825, Transport and Mobility Laboratory, École Polytechnique Fédérale de Lausanne, Switzerland.

Markov, I., Varone, S., and Bierlaire, M. (2014). Vehicle routing for a complex waste collection problem. In Proceedings of the 14th Swiss Transport Research Conference (STRC), Ascona, Switzerland.

Markov, I., Varone, S., and Bierlaire, M. (2016b). Integrating a heterogeneous fixed fleet and a flexible assignment of destination depots in the waste collection VRP with intermediate facilities. Transportation Research Part B: Methodological, 84:256-273.

Mendoza, J. E., Castanier, B., Guéret, C., Medaglia, A. L., and Velasco, N. (2011). Constructive heuristics for the multicompartment vehicle routing problem with stochastic demands. Transportation Science, $45(3): 346-363$.

Mes, M. (2012). Using simulation to assess the opportunities of dynamic waste collection. In Bangsow, S., editor, Use Cases of Discrete Event Simulation, pages 277-307. Springer Berlin Heidelberg.

Mes, M., Schutten, M., and Rivera, A. P. (2014). Inventory routing for dynamic waste collection. Waste Management, 34(9):1564-1576.

Minoux, M. (2009). Robust linear programming with right-hand-side uncertainty, duality and applications. In Floudas, C. A. and Pardalos, P. M., editors, Encyclopedia of Optimization, 2nd edition, pages 33173327. Springer.

Moraes, L. A. and Faria, L. F. (2016). A stochastic programming approach to liquified natural gas planning. Pesquisa Operacional, 36:151-165.

Niakan, F. and Rahimi, M. (2015). A multi-objective healthcare inventory routing problem; a fuzzy possibilistic approach. Transportation Research Part E: Logistics and Transportation Review, 80:74-94.

Nolz, P. C., Absi, N., and Feillet, D. (2011). Optimization of infectious medical waste collection using RFID. In Böse, J. W., Hu, H., Jahn, C., Shi, X., Stahlbock, R., and Voß, S., editors, Computational Logistics, volume 6971 of Lecture Notes in Computer Science, pages 86-100. Springer Berlin Heidelberg.

Nolz, P. C., Absi, N., and Feillet, D. (2014a). A bi-objective inventory routing problem for sustainable waste management under uncertainty. Journal of Multi-Criteria Decision Analysis, 21(5-6):299-314.

Nolz, P. C., Absi, N., and Feillet, D. (2014b). A stochastic inventory routing problem for infectious medical waste collection. Networks, 63(1):82-95. 
Papageorgiou, D. J., Nemhauser, G. L., Sokol, J., Cheon, M.-S., and Keha, A. B. (2014). MIRPLib-A library of maritime inventory routing problem instances: Survey, core model, and benchmark results. European Journal of Operational Research, 235(2):350-366.

Pillac, V., Gendreau, M., Guéret, C., and Medaglia, A. L. (2013). A review of dynamic vehicle routing problems. European Journal of Operational Research, 225(1):1-11.

Poggi, M. and Uchoa, E. (2014). Chapter 3: New exact algorithms for the capacitated vehicle routing problem. In Toth, P. and Vigo, D., editors, Vehicle Routing: Problems, Methods, and Applications, Second Edition. SIAM.

Powell, W. B. (2011). Approximate Dynamic Programming: Solving the Curses of Dimensionality, Second Edition. John Wiley \& Sons.

Rahimi, M., Baboli, A., and Rekik, Y. (2017). Multi-objective inventory routing problem: A stochastic model to consider profit, service level and green criteria. Transportation Research Part E: Logistics and Transportation Review, 101:59-83.

Ritzinger, U., Puchinger, J., and Hartl, R. F. (2016). A survey on dynamic and stochastic vehicle routing problems. International Journal of Production Research, 54(1):215-231.

Rossi, R., Tomasella, M., Martin-Barragan, B., Embley, T., Walsh, C., and Langston, M. (2017). The dynamic bowser routing problem. Technical report, Business School, University of Edinburgh, UK.

Semet, F., Toth, P., and Vigo, D. (2014). Chapter 2: Classical exact algorithms for the capacitated vehicle routing problem. In Toth, P. and Vigo, D., editors, Vehicle Routing: Problems, Methods, and Applications, Second Edition. SIAM.

Shi, Y., Boudouh, T., and Grunder, O. (2017). A hybrid genetic algorithm for a home health care routing problem with time window and fuzzy demand. Expert Systems with Applications, 72:160-176.

SITG (2017). Points de collecte des déchets. In Système d'Information du Territoire à Genève. http://ge.ch/sitg/geodata/SITG/OPENDATA/8438/SHP_GOL_DECHETTERIE.zip, Accessed on February 8, 2017.

Solyalı, O., Cordeau, J.-F., and Laporte, G. (2012). Robust inventory routing under demand uncertainty. Transportation Science, 46(3):327-340.

Soysal, M. (2016). Closed-loop inventory routing problem for returnable transport items. Transportation Research Part D: Transport and Environment, 48:31-45.

Soyster, A. L. (1973). Technical note-Convex programming with set-inclusive constraints and applications to inexact linear programming. Operations Research, 21(5):1154-1157.

Sungur, I., Ordóñez, F., and Dessouky, M. (2008). A robust optimization approach for the capacitated vehicle routing problem with demand uncertainty. IIE Transactions, 40(5):509-523.

Trudeau, P. and Dror, M. (1992). Stochastic inventory routing: Route design with stockouts and route failures. Transportation Science, 26(3):171-184.

Yu, Y. (2009). Stochastic ship fleet routing with inventory Limits. PhD thesis, University of Edinburgh, UK.

Zhalechian, M., Tavakkoli-Moghaddam, R., Zahiri, B., and Mohammadi, M. (2016). Sustainable design of a closed-loop location-routing-inventory supply chain network under mixed uncertainty. Transportation Research Part E: Logistics and Transportation Review, 89:182-214.

Zheng, S. and Chen, S. (2016). Fleet replacement decisions under demand and fuel price uncertainties. Transportation Research Part D: Transport and Environment. Available online. 